\title{
A model of radiative and conductive energy transfer in planetary regoliths
}

\author{
Bruce Hapke \\ Department of Geology and Planetary Science, University of Pittsburgh, Pittsburgh, Pennsylvania
}

\begin{abstract}
The thermal regime in planetary regoliths involves three processes: propagation of visible radiation, propagation of thermal radiation, and thermal conduction. The equations of radiative transfer and heat conduction are formulated for particulate media composed of anisotropically scattering particles. Although the equations are time dependent, only steady state problems are considered in this paper. Using the two-stream approximation, solutions are obtained for two cases: a layer of powder heated from below and an infinitely thick regolith illuminated by visible radiation. Radiative conductivity, subsurface temperature gradients, and the solid state greenhouse effect all appear intrinsically in the solutions without ad hoc additions. Although the equations are nonlinear, approximate analytic solutions that are accurate to a few percent are obtained. Analytic expressions are given for the temperature distribution, the optical and thermal radiance distributions, the hemispherical albedo, the hemispherical emissivity, and the directional emissivity. Additional applications of the new model to three problems of interest in planetary regoliths are presented by Hapke [this issue].
\end{abstract}

\section{Introduction}

The temperature distribution in the upper layers of the regolith of a planet is important for two reasons: this region is the one sampled by remote measurements of the thermal radiation emitted by a planet, and the temperature of these layers constitutes the boundary condition for thermal models of the deeper interior. However, few of the published thermal models for this region treat radiation adequately. The classical analysis by Wesselink [1948] neglected both optical and thermal radiation except as a boundary condition, as did Spencer [1990] in his important discussion of the effects of surface roughness. Usually, when thermal radiation is included its only effect is assumed to be a term proportional to $T^{3}$, where $T$ is the absolute temperature [e.g., Henderson and Jakosky, 1994] (however, notable exceptions are papers by Conel [1969] and B. Henderson and B. Jakosky (Near-surface thermal gradients and mid-IR spectra: A new model including scattering and application to real data, submitted to Journal of Geophysical Research, 1 [1995]). This assumption is a good approximation for the deep interior, where thermodyriamic equilibrium prevails, but is incorrect near the surface, where the radiation escapes to space and is not in equilibrium with the medium. Although the solid state greenhouse model of Brown and Matson [1987] allows visible sunlight to penetrate into the medium, it does not include the effects of scattering; hence the discussions in their paper are incomplete. This paper uses the heat equation and the equation of radiative transfer to describe the flow of energy within a particulate medium. The effects of both visible and thermal radiation on the temperature distribution are included, in addition to ordinary solid state conductivity. Thus radiative conductivity and the solid state greenhouse effect appear intrinsically in the model.

In section 2 the basic equations that govern the thermal and

Copyright 1996 by the American Geophysical Union.

Paper number 96JE00917.

0148-0227/96/96JE-00917\$09.00 radiative conditions are introduced. For maximum generality, the quantities that appear in these equations are then reduced to dimensionless form. The time-independent equations are solved in section 3 for a particulate medium of finite thickness whose lower boundary is held at a fixed temperature, and in section 4 for a semi-infinite medium in equilibrium with incident sunlight. The distribution of temperature and radiance with depth is calculated for several representative cases. As with my previous radiative models, quantitative analytical solutions are derived which, although approximate, retain the essential physics and are sufficiently accurate for most planetary remote sensing applications.

Only time-independent problems are treated in this paper. Although the differential equations for the general timedependent case are given, their solutions will be considered in a separate paper (B. Hapke, manuscript in preparation, 1996). Using this model, three problems of interest in planetary remote sensing are treated by Hapke [this issue].

\section{Basic Equations}

\subsection{Assumptions and Notation}

The system that will be considered in this paper is a semiinfinite, horizontally stratified, particulate medium in a vacuum, in equilibrium either with sunlight incident on its upper surface or with a lower boundary held at a fixed temperature. The particles of the medium are assumed to be irregular in shape, randomly positioned and oriented, with their light scattering and thermal properties independent of depth. An exact, quantitative description of the propagation of radiation through such a medium is beyond present capabilities, even with the aid of modern high-speed computers. Hence a number of simplifying assumptions are necessary. The most important assumption is that the radiation fields in the medium can be described by the equation of radiative transfer. In principle, this equation applies only to radiation propagating through a continuous medium whose elements do not interact coher- 


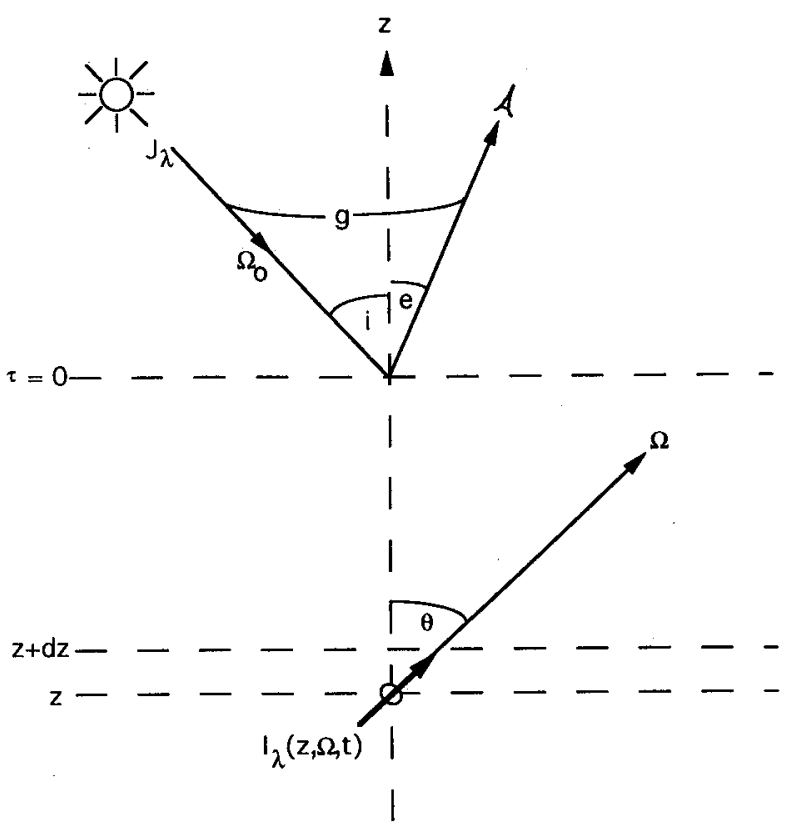

Figure 1. Schematic diagram of the geometry of the model.

ently. However, Hapke [1981, 1993a] has argued that in a random particulate medium most coherent effects are negligible and has shown that solutions of the equation provide reasonable descriptions of the bidirectional reflectances of planetary regoliths in the visible. Goguen [1996] has compared exact and approximate solutions of the radiative transfer equation with measurements on a medium of microspheres whose scattering properties are known and found reasonable agreement. Moersch and Christensen [1995] also found that models based on the radiative transfer equation predicted the spectral emissivity of powders reasonably well. Hence this assumption has empirical support.

The notation and nomenclature used in this paper are similar to those of Hapke [1993a], but with necessary additions and modifications. For convenience and reference, quantities are listed in the notation section at the end of the paper. A subscript $\lambda$ denotes that a quantity is wavelength-dependent, while a subscript $v$ or $T$ denotes that the quantity is averaged over the visible or thermal infrared spectral region, respectively; an asterisk denotes a reduced quantity.

The reduced quantities are defined as follows:

$$
\begin{gathered}
E_{\lambda}^{*}(z)=\zeta_{\lambda}^{2} E_{\lambda}(z), \quad E_{t}^{*}(z)=\zeta_{\nu}^{2} E_{\nu}(z), \\
E_{T}^{*}(z)=\zeta_{T}^{2} E_{T}(z) . \\
q=\left(\sigma_{0} / \pi\right) T_{r}^{4} / E_{T}^{*} k T_{r}=\sigma_{0} T_{r}^{3} / \pi E_{T}^{*} k
\end{gathered}
$$

(see below).

$$
R=d \tau_{v}^{*} / d \tau_{T}^{*}=E_{v}^{*} / E_{T}^{*}=E_{y} \zeta_{v}^{2} / E_{T} \zeta_{T}^{2}
$$

(see below).

$T_{r}$ is the reference temperature that characterizes the particular system.

$$
T^{*}\left(\tau_{T}^{*}, t^{*}\right)=T\left(\tau_{T}, t\right) / T_{r}
$$

The diffusion time is $t_{D}=\rho C / E_{T}^{* 2} k$

$$
t^{*}=t / t_{D}
$$

$$
\begin{gathered}
w_{\lambda}^{*}=\left(1-\beta_{\lambda}\right) w_{\lambda} / \zeta_{\lambda v}^{2}, \quad w_{v}^{*}=\left(1-\beta_{v}\right) w_{v} / \zeta_{v}^{2}, \\
w_{T}^{*}=\left(1-\beta_{T}\right) w_{T} / \zeta_{T}^{2} . \\
\varphi_{v}^{*}\left(\tau_{v}^{*}, t^{*}\right)=\varphi_{v}\left(\tau_{v}, t\right) / J_{v}, \quad \varphi_{T}^{*}\left(\tau_{T}^{*}, t^{*}\right)=\varphi_{T}(\tau, t) /\left(\sigma_{0} T_{r}^{4} / \pi\right) . \\
\Delta \varphi_{v}^{*}\left(\tau_{v}^{*}, t^{*}\right)=\Delta \varphi_{v}\left(\tau_{v}, t\right) / J_{v}, \\
\Delta \varphi_{T}^{*}\left(\tau_{T}^{*}, t^{*}\right)=\Delta \varphi_{T}(\tau, t) /\left(\sigma_{0} T_{r}^{4} / \pi\right) . \\
\gamma_{\lambda}^{*}=\left(1-w_{\lambda}^{*}\right)^{1 / 2}=\gamma_{\lambda} / \zeta_{\lambda}, \quad \gamma_{v}^{*}=\left(1-w_{v}^{*}\right)^{1 / 2}=\gamma_{v} / \zeta_{v}, \\
\gamma_{T}^{*}=\left(1-w_{T}^{*}\right)^{1 / 2}=\gamma_{T} / \zeta_{T} . \\
\mu_{\lambda}^{*}=\zeta_{\lambda}^{2} \mu, \quad \mu_{v}^{*}=\zeta_{v}^{2} \mu, \quad \mu_{T}^{*}=\zeta_{T}^{2} \mu . \\
\mu_{0 \lambda}^{*}=\zeta_{\lambda}^{2} \mu_{0}, \quad \mu_{0 v}^{*}=\zeta_{v}^{2} \mu_{0}, \quad \mu_{0 T}^{*}=\zeta_{T}^{2} \mu_{0} . \\
d \tau_{\lambda}^{*}=\zeta_{\lambda}^{2} d \tau, \quad d \tau_{v}^{*}=\zeta_{v}^{2} d t_{v}, \quad d \tau_{T}^{*}=\zeta_{T}^{2} d \tau_{T} .
\end{gathered}
$$

If visible irradiance is incident, choose $T_{r}$ as the blackbody radiative equilibrium temperature, $T_{r}=\left(\pi J_{v} / \sigma_{0}\right)^{1 / 4}$, so that $q=J_{v} / E_{T}^{*} k T_{r}=\sigma_{0} J_{v}^{3 / 4} / \pi E_{T}^{*} k$; if $J_{v}$ is the solar irradiance, then $q=\sigma_{0} \mathscr{S}^{3 / 4} / \pi E_{T}^{*} k D^{3 / 2}$, where $\mathscr{S}$ is the solar constant and $D$ is the distance to the Sun in AU. If the particle properties do not change with depth, so that $E_{v}^{*} / E_{T}^{*}$ is constant, then $R=\tau_{v}^{*} / \tau_{T}^{*}$ at all optical depths. It is usually more convenient to choose $\tau_{T}^{*}$ as the depth variable, but $\tau_{T}^{*}$ and $\tau_{v}^{*}$ may be readily interconverted using $R$.

\subsection{Equations}

The geometry is shown in Figure 1. The wavelengthdependent equation of radiative transfer for light that has been scattered one or more times within the medium, and including both external and thermal emission sources, is

$$
\begin{aligned}
\cos \vartheta & \frac{\partial I_{\lambda}(z, \Omega, t)}{\partial z}=-E_{\lambda}(z) I_{\lambda}(z, \Omega, t) \\
& +\frac{1}{4 \pi} \int_{4 \pi} I_{\lambda}\left(z, \Omega^{\prime}, t\right) G_{\lambda}\left(z, \Omega^{\prime}, \Omega\right) d \Omega^{\prime} \\
& +\frac{J_{\lambda} F(t)}{4 \pi} G_{\lambda}\left(z, \Omega_{0}, \Omega\right) \\
& \cdot \exp \left[-\frac{1}{\mu_{0}(t)} \int_{z}^{\infty} E_{\lambda}\left(z^{\prime}\right) d z^{\prime}\right]+\frac{K_{\lambda}}{\pi} U_{\lambda}[T(z, t)],
\end{aligned}
$$

where $I_{\lambda}(z, \Omega, t)$ is the spectral radiance (power per unit area per unit steradian per unit wavelength interval at elevation $z$ traveling in direction $\Omega$ at time $t$ and wavelength $\lambda$ ), $\vartheta$ is the angle between $\Omega$ and the $z$ axis, $E_{\lambda}(z)$ is the volume extinction coefficient at elevation $z$ and wavelength $\lambda, G_{\lambda}\left(z, \Omega^{\prime}, \Omega\right)$ is the volume coefficient at location $z$ and wavelength $\lambda$ for scattering radiance from direction $\pi-\Omega^{\prime}$ into direction $\Omega, J_{\lambda}$ is the spectral irradiance from the source, $F(t)$ describes the time dependence of the source irradiance, $\mu_{0}(t)$ is the cosine of the angle of incidence, $K_{\lambda}(z)$ is the spectral volume absorption coefficient at location $z$ and wavelength $\lambda$ (by Kirchhoff's law, equal to the emissivity), and $U_{\lambda}(T)$ is the Planck function for the power emitted per unit area per unit wavelength interval from a blackbody at temperature $T$,

$$
U_{\lambda}(T(z, t)]=\left[2 \pi h_{0} c_{0} / \lambda^{5}\right]\left[e^{h_{0} c_{0} / k_{1} \mid \lambda T(z, t)}-1\right]^{-1},
$$


where $h_{0}$ is Planck's constant, $c_{0}$ is the speed of light in vacuum, $k_{0}$ is Boltzmann's constant, and $T(z, t)$ is the absolute temperature.

In addition to the radiative transfer equation, the temperature must also satisfy the heat equation

$$
\begin{aligned}
& \rho C \frac{\partial T(z, t)}{\partial t}=\frac{\partial}{\partial z}\left[k \frac{\partial T(z, t)}{\partial z}\right] \\
& +\int_{\lambda} K_{\lambda}(z) J_{\lambda} F(t) \exp \left[-\frac{1}{\mu_{0}(t)} \int_{z}^{\infty} E_{\lambda}\left(z^{\prime}\right) d z^{\prime}\right] d \lambda \\
& +\int_{\lambda} K_{\lambda}(z) \int_{4 \pi} I_{\lambda}(z, \Omega, t) d \Omega d \lambda \\
& -\int_{\lambda} K_{\lambda}(z) \int_{4 \pi} \frac{1}{\pi} U_{\lambda}[T(z, t)] d \Omega d \lambda,
\end{aligned}
$$

where $\rho$ is the bulk density, $C$ is the specific heat per unit mass, $k$ is the thermal conductivity, and $K_{\lambda}(z)$ is the spectral volume absorption coefficient. The parameter $k$ is the intrinsic, solidstate thermal conductivity of the medium only and does not include radiative heat transfer, which is specifically accounted for by the thermal radiance.

The term on the left-hand side of (3) describes the rate of change of heat content per unit volume in a layer of thickness $d z$ at depth $z$. The first term on the right-hand side of (3) is the difference between the heat conducted into a layer at depth $z$ and the heat conducted out. The second term on the right describes the source of heat due to unscattered light from the source that has penetrated to $z$ and been absorbed there. The third term is the scattered radiance absorbed at depth $z$, while the fourth term describes the loss of heat by thermal radiation from the particles in the element.

The heat equation is coupled to the radiative transfer equation only through integrals over wavelength, rather than the spectrally resolved radiance. In a medium illuminated by a source of visible light, such as the Sun, the radiance is appreciable in only two spectral regions, the visible (including the near-UV, visible, and near-IR) and the thermal IR, and the temperature distribution is determined by the integrals of the spectral radiance over those two regions. In many problems of interest the two wavelength intervals are sufficiently separated that the area of overlap contributes little to the integrals. This assumption will be made here. Note that this assumption does not preclude treating regions of the spectrum where both visible and thermal radiation are important in the spectral radiative transfer equation [e.q., Hapke, 1993a, b], only that such regions contribute little to the integrals over wavelength.

The system of coupled partial differential equations will be solved by the following procedure. The equation of radiative transfer will be converted into two differential equations by integrating over the visible and thermal wavelength regions separately. Since the resulting equation governing the visible radiation does not contain the temperature, it may be solved to give the visible radiance as a function of $z$. This solution will be inserted into the heat equation. The coupled heat equation and thermal radiative transfer equation will then be solved simultaneously to give the temperature and thermal radiance distributions. Once the temperature is known, it can be inserted into the source function $U_{\lambda}(T)$ in the spectral radiative transfer equation (1), which can be solved to give the radiance at any wavelength.

The equations will be further simplified by introducing wavelength-averaged parameters as follows. Integrating the radiative transfer equation over the visible portion of the spectrum gives

$$
\begin{aligned}
\cos \vartheta & \frac{\partial \int_{\text {visible }} I_{\lambda}(z, \Omega, t) d \lambda}{\partial z} \\
& =-\int_{\text {visible }} E_{\lambda}(z) I_{\lambda}(z, \Omega, t) d \lambda+\cdots
\end{aligned}
$$

Since the visible radiance consists of scattered light from the source, it has a spectral distribution similar to that of the source $J_{\lambda}$ but modified by departures from a gray body caused by absorption bands in the medium. In order to make the equations tractable, it will be assumed that to a sufficient approximation, the various parameters in the visible radiative transfer equation may be replaced by their averages over the visible portion of the spectrum weighted by the spectrum of the source. Thus, for example, the first term on the right-hand side of the integrated equation can be written

$$
\int_{\text {visible }} E_{\lambda}(z) I_{\lambda}(z, \Omega, t) d \lambda=E_{\nu}(z) \int_{\text {visible }} I_{\lambda}(z, \Omega, t) d \lambda
$$

where $E_{y}(z)$ is the average visible extinction coefficient,

$$
\begin{aligned}
E_{v}(z) & =\int_{\text {visible }} E_{\lambda}(z) I_{\lambda}(z, \Omega, t) d \lambda / \int_{\text {visible }} I_{\lambda}(z, \Omega, t) d \lambda \\
& \simeq \int_{\lambda} E_{\lambda}(z) J_{\lambda} d \lambda / \int_{\lambda} J_{\lambda} d \lambda
\end{aligned}
$$

Similarly, the IR radiance at any location in the medium has a spectral distribution similar to the Planck function of a blackbody at the local temperature, modified by any absorption bands in the medium at thermal IR wavelengths. Again, in order to make the problem tractable, it will be assumed that the parameters in the IR radiative transfer equation may, to sufficient approximation, be replaced by their averages over the IR portion of the spectrum weighted by the Planck function of a body at a reference temperature $T$, that is characteristic of the system. For instance, in a medium illuminated by visible radiation, $T_{r}$ might be the radiative equilibrium temperature, or if no visible source is present, as in a laboratory experiment, $T_{r}$ might be the temperature of the source supplying heat to the medium. Thus, when the radiative transfer equation is integrated over the thermal IR, the extinction coefficient is replaced by its average thermal value:

$$
\begin{aligned}
E_{T}(z) & =\int_{\text {thermal }} E_{\lambda}(z) I_{\lambda}(z, \Omega, t) d \lambda / \int_{\text {thermal }} I_{\lambda}(z, \Omega, t) d \lambda \\
& =\int_{\text {thermal }} E_{\lambda}(z) U_{\lambda}\left(T_{r}\right) d \lambda / \int_{\text {thermal }} U_{\lambda}\left(T_{r}\right) d \lambda .
\end{aligned}
$$

Other parameters that appear in the wavelength-integrated visible and thermal-IR equations of radiative transfer will be 
replaced by their similarly averaged values. This procedure will not introduce serious errors if the reflectance spectrum of the medium is relatively bland in the visible and thermal IR near the maxima of $J_{\lambda}$ and $U_{\lambda}\left(T_{r}\right)$. However, if broad spectral features of large contrast are present there, the spectrum of $I_{\lambda}(z, \Omega, t)$ could be appreciably different from $J_{\lambda}$ or $U_{\lambda}\left(T_{r}\right)$, which could cause wavelength-averaged parameters to be unrepresentative of their true values.

In this paper it will be assumed that wavelength averaging each parameter, as described above, is valid. Let $I_{v}(z, \Omega, t)$ and $I_{T}(z, \Omega, t)$ be the wavelength-integrated visible and thermal-IR radiances, respectively. Let a subscript $v$ on any other quantity denote the average value of that quantity weighted by the spectrum of the source of visible radiation integrated over wavelength. Let a subscript $T$ on any other quantity denote the average value weighted by the Planck function of a blackbody at some temperature that characterizes the system integrated over wavelength.

Then the equation of radiative transfer for visible radiation becomes

$$
\begin{aligned}
\cos \vartheta & \frac{\partial I_{v}(z, \Omega, t)}{\partial z}=-E_{v}(z) I_{v}(z, \Omega, t) \\
& +\frac{1}{4 \pi} \int_{4 \pi} I_{v}\left(z, \Omega^{\prime}, t\right) G_{v}\left(z, \Omega^{\prime}, \Omega\right) d \Omega^{\prime} \\
& +J_{v} F(t) \frac{1}{4 \pi} G_{v}\left(z, \Omega_{0}, \Omega\right) \\
& \cdot \exp \left(-\frac{1}{\mu_{0}(t)} \int_{z}^{\infty} E_{v}\left(z^{\prime}\right) d z^{\prime}\right) .
\end{aligned}
$$

Let $\tau_{v}=\int_{z}^{\infty} E_{v}\left(z^{\prime}\right) d z^{\prime}$ be the optical depth for visible light. Putting $G_{v}\left(z, \Omega^{\prime}, \Omega\right)=S_{v}(z) p_{y}\left(z, \Omega^{\prime}, \Omega\right), w_{v}(z)=$ $S_{v}(z) / E_{v}(z)$, dividing by $E_{v}(z)$, and making $\tau_{v}$ the spatial variable, this equation can be written in the following form:

$$
\begin{aligned}
\cos \vartheta & \frac{\partial I_{v}\left(\tau_{v}, \Omega, t\right)}{\partial \tau_{v}}=-I_{v}\left(\tau_{v}, \Omega, t\right) \\
& +\frac{w_{v}\left(\tau_{v}\right)}{4 \pi} \int_{4 \pi} I_{v}\left(\tau_{v}, \Omega^{\prime}, t\right) p_{v}\left(\tau_{v}, g^{\prime}\right) d \Omega^{\prime} \\
& +J_{v} F(t) \frac{w_{v}\left(\tau_{v}\right)}{4 \pi} p_{v}\left(\tau_{v}, g_{0}\right) e^{-\tau_{,} / \mu_{0}(t)},
\end{aligned}
$$

where $g^{\prime}$ is the angle between $\pi-\Omega^{\prime}$ and $\Omega, g_{0}$ is the angle between $\Omega_{0}$ and $\Omega, S_{z}(z)$ is the visible scattering coefficient, $p_{y}\left(z, \Omega^{\prime}, \Omega\right)$ is the visible phase function, and $w_{v}(z)$ is the visible single scattering albedo.

Since

$$
\int_{T} U_{\lambda}(T) d \lambda=\sigma_{0} T^{4}
$$

where $\sigma_{0}$ is the Stefan-Boltzmann constant, the wavelengthintegrated equation of radiative transfer for thermal radiation is

$\cos \vartheta \frac{\partial I_{T}(z, \Omega, t)}{\partial z}=-E_{T}(z) I_{T}(z, \Omega, t)$

$$
\begin{aligned}
& +\frac{1}{4 \pi} \int_{4 \pi} I_{T}\left(z, \Omega^{\prime}, t\right) G_{T}\left(z, \Omega^{\prime}, \Omega\right) d \Omega^{\prime} \\
& +K_{T}(z) \frac{\sigma_{0}}{\pi} T^{4}(z, t) .
\end{aligned}
$$

Let $\tau_{T}=\int_{z}^{\infty} E_{T}\left(z^{\prime}\right) d z^{\prime}$ be the optical depth for IR radiation. Putting $G_{T}\left(z, \Omega^{\prime}, \Omega\right)=S_{T}(z) p_{T}\left(z, \Omega^{\prime}, \Omega\right), w_{T}(z)=$ $S_{T}(z) / E_{T}(z), \mathscr{E}_{T}(z)=K_{T}(z) / E_{T}(z)$; dividing by $E_{T}(z)$; and making $\tau_{T}$ the spatial variable, this equation can be written in the form

$$
\begin{aligned}
\cos \vartheta & \frac{\partial I_{T}\left(\tau_{T}, \Omega, t\right)}{\partial \tau_{T}}=-I_{T}\left(\tau_{T}, \Omega, t\right)+\frac{w_{T}\left(\tau_{T}\right)}{4 \pi} \\
& \cdot \int_{4 \pi} I_{T}\left(\tau_{T}, \Omega^{\prime}, t\right) p_{T}\left(\tau_{T}, g^{\prime}\right) d \Omega^{\prime} \\
& +\mathscr{E}_{T}\left(\tau_{T}\right) \frac{\sigma_{0}}{\pi} T^{4}\left(\tau_{T}, t\right),
\end{aligned}
$$

where $S_{T}(z)$ is the thermal scattering coefficient, $p_{T}\left(z, \Omega^{\prime}\right.$, $\Omega)$ is the thermal phase function, $w_{T}(z)$ is the thermal single scattering albedo, and $\mathscr{E}_{T}(z)$ is the volume emissivity.

Similarly, the heat equation is

$$
\begin{aligned}
& \rho C \frac{\partial T(z, t)}{\partial t}=\frac{\partial}{\partial z}\left[k \frac{\partial T(z, t)}{\partial z}\right]+K_{T}(z) \int_{4 \pi} I_{T}(z, \Omega, t) d \Omega \\
& \quad-K_{T}(z) \int_{4 \pi} \frac{\sigma_{0}}{\pi} T^{4}(z, t) d \Omega \\
& +K_{v}(z) J_{v} F(t) \exp \left(-\frac{1}{\mu_{0}(t)} \int_{z}^{\infty} E_{v}\left(z^{\prime}\right) d z^{\prime}\right) \\
& +K_{v}(z) \int_{4 \pi} I_{v}(z, \Omega, t) d \Omega,
\end{aligned}
$$

which can be written

$$
\begin{aligned}
& \rho_{c} \frac{\partial T(z, t)}{\partial t}=\frac{\partial}{\partial z}\left[k \frac{\partial T(z, t)}{\partial z}\right]+K_{T}(z) \int_{4 \pi} I_{T}(z, \Omega, t) d \Omega \\
& \left.\quad-K_{T}(z) \int_{4 \pi} \frac{\sigma_{0}}{\pi} T^{4}(z, t)\right] d \Omega+K_{v}(z) J F(t) e^{-\tau_{v} / \mu} \\
& \quad+K_{v}(z) \int_{4 \pi} I_{v}(z, \Omega, t) d \Omega .
\end{aligned}
$$

Equations (4), (6), and (7) are the fundamental equations to be solved. It will be assumed that all of the quantities in these equations, except the radiances and temperature, are independent of $z$ and $T$. This system of equations will then be solved using the two-stream method. In this approximation, the radiative transfer equation is integrated with respect to solid angle separately over the upward going $(0<\vartheta<\pi / 2)$ and downward going $(\pi / 2<\vartheta<\pi)$ hemispheres, and each quantity in each integral is replaced by its average value in that hemisphere. See 
Hapke [1993a] for details. Anisotropic scattering is taken into account by the hemispherical asymmetry parameter $\beta$. This quantity is defined such that a fraction $(1+\beta) / 2$ of the light incidence on a particle from any direction within an upward going or downward going hemisphere is treated as scattered uniformly forward into the same hemisphere into which the light was traveling, and a fraction $(1-\beta) / 2$ treated as scattered backward uniformly into the opposite hemisphere.

Let $I_{v 1}\left(\tau_{v}, t\right)$ and $I_{v 2}\left(\tau_{v}, t\right)$ be the hemispherically averaged upward going and downward going visible radiances, respectively,

$$
\begin{gathered}
I_{v 1}\left(\tau_{v}, t\right)=\frac{1}{2 \pi} \int_{\vartheta=0}^{\pi / 2} I_{v}\left(\tau_{v}, \Omega, t\right) d \Omega \\
I_{v 2}\left(\tau_{v}, t\right)=\frac{1}{2 \pi} \int_{\vartheta=\pi / 2}^{\pi} I_{v}\left(\tau_{v}, \Omega, t\right) d \Omega .
\end{gathered}
$$

The result of hemispherical averaging of (4) for the visible radiation consists of two coupled equations:

$$
\begin{aligned}
& -\frac{1}{2} \frac{\partial I_{v 1}\left(\tau_{v}, t\right)}{\partial \tau_{v}}=-\left[1-\frac{w_{v}}{2}\left(1+\beta_{v}\right)\right] I_{v 1}\left(\tau_{v}, t\right) \\
& +\frac{w_{v}}{2}\left(1-\beta_{v}\right) I_{v 2}\left(\tau_{v}, t\right)+J_{v} F(t) \frac{w_{v}}{4 \pi}\left(1-\beta_{v}\right) e^{-\tau_{v} / \mu_{0}(t)},
\end{aligned}
$$

and

$$
\begin{aligned}
& +\frac{1}{2} \frac{\partial I_{v 2}\left(\tau_{v}, t\right)}{\partial \tau_{v}}=-\left[1-\frac{w_{v}}{2}\left(1+\beta_{v}\right)\right] I_{v 2}\left(\tau_{v}, t\right) \\
& +\frac{w_{v}}{2}\left(1-\beta_{v}\right) I_{v 1}\left(\tau_{v}, t\right)+J_{v} F(t) \frac{w_{v}}{4 \pi}\left(1+\beta_{v}\right) e^{-\tau_{v} / \mu 1(t)},
\end{aligned}
$$

where the factor of $\pm 1 / 2$ on the left-hand side of (9a) and (9b) is the average value of $\cos \vartheta$ in each hemisphere. Let

$$
\begin{gathered}
\varphi_{v}\left(\tau_{v}, t\right)=\left[I_{v 1}\left(\tau_{v}, t\right)+I_{v 2}\left(\tau_{v}, t\right)\right] / 2 ; \\
\Delta \varphi_{\nu}\left(\tau_{v}, t\right)=\left[I_{v 1}\left(\tau_{v}, t\right)-I_{z 2}\left(\tau_{v}, t\right)\right] / 2
\end{gathered}
$$

$\varphi_{v}\left(\tau_{v}, t\right)$ is the spherically averaged radiance in the medium at optical depth $\tau_{v}$, and time $t$. By alternatively adding and subtracting (9a) and (9b), they can be put into the form

$$
-\frac{1}{2} \frac{\partial \Delta \varphi_{v}\left(\tau_{v}, t\right)}{\partial \tau_{v}}=-\left(1-w_{v}\right) \varphi_{v}\left(\tau_{v}, t\right)+J_{y} F(t) \frac{w_{v}}{4 \pi} e^{-\tau_{v} / \mu_{0}(t)}
$$

and

$$
\begin{aligned}
-\frac{1}{2} \frac{\partial \varphi_{v}\left(\tau_{v}, t\right)}{\partial \tau_{v}}= & -\left(1-\beta_{v} w_{v}\right) \Delta \varphi_{v}\left(\varphi_{v}, t\right) \\
& -J_{v} F(t) \frac{w_{v}}{4 \pi} \beta_{v} e^{-\tau_{v} / \mu_{v}(t)}
\end{aligned}
$$

Let $\gamma_{v}=\left(1-w_{v}\right)^{1 / 2}$ and $\zeta_{v}=\left(1-\beta_{v} w_{v}\right)^{1 / 2}$. Then (11b) can be solved for $\Delta \varphi_{\gamma}$,

$\Delta \varphi_{v}\left(\tau_{v}, t\right)=\frac{1}{2 \zeta_{v}^{2}} \frac{\partial \varphi_{v}\left(\tau_{v}, t\right)}{\partial \tau_{v}}-J_{v} F(t) \frac{w_{v}}{4 \pi} \frac{\beta_{v}}{\zeta_{v}^{2}} e^{-\tau_{v} / \mu_{0}(t)}$

Differentiating (12a) with respect to $\tau_{v}$, and substituting the result into (11a) gives $\frac{1}{4} \frac{\partial^{2} \varphi_{v}\left(\tau_{v}, t\right)}{\partial \tau_{v}^{2}}=\zeta_{v}^{2} \gamma_{v}^{2} \varphi_{v}\left(\tau_{v}, t\right)-J_{v} F(t) \frac{w_{v}}{4 \pi}\left(\zeta_{v}^{2}+\frac{\beta_{v}}{2 \mu_{0}}\right) e^{-\tau_{v} / \mu_{0}(t)}$

Similarly, by letting

$$
\begin{gathered}
I_{T 1}\left(\tau_{T}, t\right)=\frac{1}{2 \pi} \int_{\vartheta=0}^{\pi / 2} I_{T}\left(\tau_{T}, \Omega, t\right) d \Omega, \\
I_{T 2}\left(\tau_{T}, t\right)=\frac{1}{2 \pi} \int_{\vartheta=\pi / 2}^{\pi} I_{T}\left(\tau_{T}, \Omega, t\right) d \Omega, \\
\varphi_{T}\left(\tau_{T}, t\right)=\left[I_{T 1}\left(\tau_{T}, t\right)+I_{T 2}\left(\tau_{T}, t\right)\right] / 2, \\
\left.\Delta \varphi_{T}\left(\tau_{T}, t\right)=\left[I_{T 1}\left(\tau_{T}, t\right)\right]-I_{T 2}\left(\tau_{T}, t\right)\right] / 2,
\end{gathered}
$$

and assuming that the particles of the medium radiate isotropically, the IR radiative transfer equation can be put into the form

$$
\begin{gathered}
-\frac{1}{2} \frac{\partial \Delta \varphi_{T}\left(\tau_{T}, t\right)}{\partial \tau_{T}}=-\left(1-w_{T}\right) \varphi_{T}\left(\tau_{T}, t\right)+\mathscr{E}_{T} \frac{\sigma_{0}}{\pi} T^{4}\left(\tau_{T}, t\right) \\
-\frac{1}{2} \frac{\partial \varphi_{T}\left(\tau_{T}, t\right)}{\partial \tau_{T}}=-\left(1-\beta_{T} w_{T}\right) \Delta \varphi_{T}\left(\tau_{T}, t\right),
\end{gathered}
$$

Similarly, letting $\gamma_{T}=\left(1-w_{T}\right)^{1 / 2}$ and $\zeta_{T}=\left(1-\beta_{T} w_{T}\right)^{1 / 2}$ and realizing that $\mathscr{E}_{T}=\gamma_{T}^{2}$, equations (15a) and (15b) can be put into the form

$$
\Delta \varphi_{T}\left(\tau_{T}, t\right)=\frac{1}{2 \zeta_{T}^{2}} \frac{\partial \varphi_{T}\left(\tau_{T}, t\right)}{\partial \tau_{T}},
$$

and

$\frac{1}{4} \frac{\partial^{2} \varphi_{T}\left(\tau_{T}, t\right)}{\partial \tau_{T}^{2}}=\zeta_{T}^{2} \gamma_{T}^{2} \varphi_{T}\left(\tau_{T}, t\right)-\zeta_{T}^{2} \gamma_{T}^{2} \frac{\sigma_{0}}{\pi} T^{4}\left(\tau_{T}, t\right)$

Finally, the heat equation can be written in the form

$$
\begin{aligned}
& \rho c \frac{\partial T(z, t)}{\partial t}=\frac{\partial}{\partial z}\left[k \frac{\partial T(z, t)}{\partial z}\right] \\
& \quad+E_{T}(z) \gamma_{T}^{2} 4 \pi\left[\varphi_{T}(z, t)-\frac{\sigma_{0}}{\pi} T^{4}(z, t)\right] \\
& \quad+E_{v}(z) \gamma_{v}^{2}\left[J_{v} F(t) e^{-\tau_{v} / \mu_{1}(t)}+4 \pi \varphi_{v}(z, t)\right] .
\end{aligned}
$$

In order to solve (12), (16), and (17), six boundary conditions must be specified. Three are that the temperature and radiances must be finite everywhere, including $z \rightarrow \infty$ if the medium is infinitely thick. If the medium is of finite thickness then conditions on the lower boundary must be given (see section 3). Two more result from requiring that there be no sources of visible or IR radiance above the $\tau=0$ level, except for the collimated irradiance $J_{y}$, which is included as a source term in the equations, and is not a boundary condition. This requires

$$
I_{v 2}(0, t)=0
$$

or

$$
\varphi_{\nu}(0, t)=\Delta \varphi_{\nu}(0, t)
$$

and 


$$
I_{T_{2}}(0, t)=0
$$

or

$$
\varphi_{T}(0, t)=\Delta \varphi_{T}(0, t) .
$$

The remaining boundary condition on $T$ comes from the requirement that the only flux of heat crossing the $\tau=0$ boundary is carried by thermal radiation and none by conduction. However, the conducted heat flux, $k \partial T / \partial z$, must be continuous across any horizontal plane, including the upper surface of the medium. Since $k=0$ above the surface, but not below it, this requires that

$$
\frac{\partial T}{\partial z}(0, t)=0
$$

as the surface is approached from below.

(These boundary conditions at the surface of the medium apply only to bodies without atmospheres. If the medium is in an atmosphere, then at the surface the heat flux conducted through the medium must be continuous with the flux conducted through the air above the surface. Also, the downward radiances $I_{v 2}$ and $I_{T 2}$ at the surface must be equal to the visible and IR radiances scattered and radiated downward by the atmosphere.)

An additional requirement on the solutions arises from physical considerations. Deep within a medium, far from any boundaries, the thermal radiation must be in equilibrium with the temperature of the material there. This requires that

$$
\varphi_{T}(z, t) \underset{z \rightarrow \infty}{\longrightarrow} \sigma_{0} T^{4}(z, t) / \pi \text {. }
$$

\subsection{Equations in Reduced Form}

In order to make these equations as general and simple as possible, it is desirable to reduce them to nondimensional form by scaling all quantities to reference quantities that characterize their magnitudes for each specific case. This has already been done in the radiation equation for the distance variable by replacing it with the optical depths $\tau_{v}$ or $\tau_{T}$, which are distance in units of the extinction lengths, $L_{E v}=E_{v}^{-1}$ or $L_{E T}=E_{T}^{-1}$. A natural quantity to use to scale the visible flux is the incident irradiance $J_{v}$. The temperature may be scaled to some reference temperature $T_{r}$ that characterizes the system, and the thermal flux may be scaled to the thermal radiance $\sigma_{0} T_{r}^{4} / \pi$ inside a cavity at that temperature. If the source of visible radiation is sunlight, it is convenient to choose $T_{r}$ as the blackbody radiative equilibrium temperature given by $J_{v}=$ $\sigma_{0} T_{r}^{4} / \pi$. Although the period of rotation $P$ of a body might be used to scale the time variable, the equations become simpler if the diffusion time $t_{D}=\rho C / \zeta_{T}^{2} E_{T}^{2} k$ is used instead.

In addition, if the particles of the medium are not isotropic scatterers, the equations may be further simplified by using similarity relations. These are reduced single scattering albedos and optical depths that convert the equations for anisotropic scatterers into expressions whose mathematical forms are as close as possible to those for isotropic scatterers.

In the remainder of the paper an asterisk will denote a reduced quantity. These are defined in section 2.1 . Substituting them into the equations formulated in 2.2 gives the reduced equations. For visible radiation, (12a), (12b), and (18) become

$\frac{1}{4} \frac{\partial^{2} \varphi_{v}^{*}\left(\tau_{v}^{*}, t^{*}\right)}{\partial \tau_{v}^{* 2}}=\gamma_{v}^{* 2} \varphi_{v}^{*}\left(\tau_{v}^{*}, t^{*}\right)$

$$
\begin{gathered}
-F(t) \frac{w_{v}^{*}}{4 \pi} \frac{1+\beta_{v} / 2 \mu_{0} v^{*}}{1-\beta_{v}} e^{-\tau_{i}^{*} / \mu_{i}^{*},\left(t^{*}\right)}, \\
\Delta \varphi_{v}^{*}\left(\tau_{v}^{*}, t^{*}\right)=\frac{1}{2} \frac{\partial \varphi_{v}^{*}\left(\tau_{v}^{*} t^{*}\right)}{\partial \tau_{v}^{*}}-F\left(t^{*}\right) \frac{w_{v}^{*}}{4 \pi} \frac{\beta_{v}}{1-\beta_{v}} e^{-\tau_{v}^{*} \mu_{v}^{*}\left(l^{*}\right)},
\end{gathered}
$$

with the boundary condition

$$
\varphi_{v}^{*}\left(0, t^{*}\right)=\frac{1}{2} \frac{\partial \varphi_{v}^{*}\left(0, t^{*}\right)}{\partial \tau_{v}^{*}}-F\left(t^{*}\right) \frac{w_{v}^{*}}{4 \pi} \frac{\beta_{v}}{1-\beta_{v}} .
$$

For thermal radiation, (16a), (16b), and (19) become

$$
\begin{gathered}
\frac{1}{4} \frac{\partial^{2} \varphi_{T}^{*}\left(\tau_{m}^{*} t^{*}\right)}{\partial \tau_{T}^{* 2}}=\gamma_{T}^{* 2}\left[\varphi_{T}^{*}\left(\tau_{T}^{*}, t^{*}\right)-T^{* 4}\left(\tau_{T}^{*}, t^{*}\right)\right] \\
\Delta \varphi_{T}^{*}\left(\tau_{T}^{*}, t^{*}\right)=\frac{1}{2} \frac{\partial \varphi_{T}^{*}\left(\tau_{T}^{*}, t^{*}\right)}{\partial \tau_{T}^{*}}
\end{gathered}
$$

with the boundary condition

$$
\varphi_{T}^{*}\left(0, t^{*}\right)=\frac{1}{2} \frac{\partial \varphi_{T}^{*}\left(0, t^{*}\right)}{\partial \tau_{T}^{*}}
$$

Arbitrarily choosing $\tau_{T}^{*}$ as the reduced spatial variable, the reduced heat transfer equation (17) becomes

$$
\begin{aligned}
& \frac{\partial T^{*}\left(\tau_{T}^{*}, t^{*}\right)}{\partial t^{*}}=\frac{\partial^{2} T^{*}\left(\tau_{T}^{*}, t^{*}\right)}{\partial \tau_{T}^{* 2}} \\
& +4 \pi q \gamma_{T}^{* 2}\left[\varphi_{T}^{*}\left(\tau_{T}^{*}, t^{*}\right)-T^{* 4}\left(\tau_{T}^{*}, t^{*}\right)\right] \\
& \quad+R q \gamma_{v}^{* 2}\left[F(t) e^{-\tau_{v}^{*} / \mu_{i}^{*}\left(t^{*}\right)}+4 \pi \varphi_{v}^{*}\left(\tau_{v}^{*}, t^{*}\right)\right],
\end{aligned}
$$

with the boundary condition

$$
\partial T^{*}\left(0, t^{*}\right) / \partial \tau_{T}^{*}=0
$$

Inserting (23a) into (24a) gives an alternate form of the reduced heat equation:

$$
\begin{aligned}
& \frac{\partial T^{*}\left(\tau_{T}^{*}, t^{*}\right)}{\partial t^{*}}=\frac{\partial^{2} T^{*}\left(\tau_{T}^{*}, t^{*}\right)}{\partial \tau_{T}^{* 2}}+\pi q \frac{\partial^{2} \varphi_{T}\left(\tau_{T}^{*}, t^{*}\right)}{\partial \tau_{T}^{* 2}} \\
& \quad+R q \gamma_{v}^{* 2}\left[F(t) e^{-\tau_{i}^{*} / \mu_{i}^{*}\left(t^{*}\right)}+4 \pi \varphi_{v}^{*}\left(\tau_{v}^{*}, t^{*}\right)\right]
\end{aligned}
$$

If there is no visible source, the last term on the right hand side of (24a) or (24c) vanishes. Other conditions on the solutions of these equations are that $\varphi_{v}^{*}, \varphi_{T}^{*}$, and $T^{*}$ must be finite and

$$
\varphi_{T}^{*}\left(\tau_{T}^{*}, t\right) \underset{\tau_{r \rightarrow \infty}^{*}}{\longrightarrow} T^{* 4}\left(\tau_{T}^{*}, t\right) .
$$

\subsection{Physical Meaning and Magnitudes of Parameters}

The diffusion time may be written $t_{D}=\rho C \Delta T L_{E T} /(k \Delta T /$ $\left.L_{E T}\right)$, where $\Delta T$ is an arbitrary, small rise in temperature and $L_{E T}=E_{T}^{-1}$ is the thermal extinction length. From this it is seen that $t_{D}$ is time to raise the temperature of a layer of thickness $L_{E T}$ by $\Delta T$ when a flux of heat $k \Delta T / L_{E T}$ is conducted through the layer.

The physical meaning of the parameter $q=\sigma_{0} T_{r}^{4} / \pi E_{T}^{*} k T_{r}$ may be seen as follows. The numerator, $\sigma_{0} T_{r}^{4} / \pi=J_{v}$, characterizes the radiant power incident in the medium, while the denominator, $E_{T}^{*} k T_{r}=k T_{r} / L_{E T}^{*}$, characterizes the flux of heat conducted by a temperature gradient of $T_{r} / L_{E T}^{*}$. Now, the first term on the right-hand side of (24a) describes, in reduced form, the net flux of heat conducted through a layer 
and the second term describes the net flux of power carried by thermal radiation through that layer. Hence $\pi q$ is a measure of the ratio of the heat conducted by radiation to that carried by solid state conduction.

To illustrate the magnitude of some of these parameters, ignore the subscripts $v$ and $T$ for the moment. Consider a simple medium composed of $N$ particles per unit volume of equant, isotropically scattering particles of average diameter $d$ and solid density $\rho_{s}$. The bulk density is $\rho \simeq N \rho_{s} \pi d^{3} / 6$, and the particle cross-sectional area is $\sigma \simeq \pi d^{2} / 4$. Hence $E=$ $n \sigma Q_{E} \simeq(3 / 2) \phi\left(Q_{E} / d\right)$, where $\phi=\rho / \rho_{s}$ is the filling factor. For particles larger than the wavelength relatively close to each other in a regolith, $Q_{E} \simeq 1$ [Hapke, 1993a] so that the extinction length is $L_{E}=E^{-1} \simeq 2 d / 3 \phi$. In loosely packed particulate media, such as the upper surface of the lunar regolith, $\phi$ can be as small as 0.3 [Carrier et al., 1973], so that $L_{E} \sim 2 d$. Apollo lunar soil samples have mean particle sizes around 50 $\mu \mathrm{m}$ [McKay et al., 1974]. Hence, for impact-generated regoliths, $L_{E} \sim 100 \mu \mathrm{m}$, and $E \sim 1 \times 10^{4} \mathrm{~m}^{-1}$.

For a medium illuminated by the Sun, $J_{v}=1360 / D^{2} \mathrm{~W}$ $\mathrm{m}^{-2}$, where $D$ is the distance to the Sun in AU, so $T_{r}=$ $\left(\pi J_{v} / \sigma_{0}\right)^{1 / 4} / \sqrt{D}=524 / \sqrt{D} \mathrm{~K}$. The temperature-independent component of the thermal conductivity of basaltic powders, including lunar soil, depends on the density, but $k \simeq$ $0.001 \mathrm{~W} \mathrm{~m}^{-1} \mathrm{~K}^{-1}$ is typical for densities around $1 \mathrm{~g} / \mathrm{cm}^{3}$ [Fountain and West, 1970]. Hence $q \simeq 0.26 \mathrm{D}^{-3 / 2}$, which ranges from 1.07 for Mercury to 0.023 for a satellite of Jupiter.

The specific heat per unit mass of lunar soil depends on the temperature, but averages about $C=550 \mathrm{~J} \mathrm{~kg}^{-1} \mathrm{~K}^{-1}$ over the temperature range from 100 to $350 \mathrm{~K}$ [Horai and Simmons, 1972]. Taking $\rho \simeq 1 \times 10^{3} \mathrm{~kg} \mathrm{~m}^{-3}$, gives $t_{D} \simeq 5.5 \mathrm{~s}$.

\section{A Medium Heated From Below}

\subsection{Solution}

The first solution that will be obtained is for a system consisting of a thick layer of powder in vacuum lying on a plate held at constant temperature $T_{p}$, and radiating into cold space from its upper surface. This might represent the situation in a laboratory measurement of emissivity where the surface of the sample sees only other surfaces at temperatures close to absolute zero.

For this problem, $d T^{*} / d t^{*}=0, J_{v}=0, \varphi_{v}^{*}=0$, and $q=$ $\sigma_{0} T_{r}^{3} / \pi E_{T}^{*} k$. Choose the reference temperature $T_{r}$ to be the temperature of the heated bottom plate $T_{p}$. Let the emissivity of this plate be $\varepsilon_{p}$ and the optical thickness of the powder at the plate be $\tau_{T p}$, so that its reduced optical thickness is $\tau_{T_{p}}^{*}=$ $\zeta_{T}^{2} \tau_{T p}$. Then equation $(24 \mathrm{c})$ is

$$
\frac{d^{2} T^{*}\left(\tau_{T}^{*}\right)}{d \tau_{T}^{* 2}}+\pi q \frac{d^{2} \varphi_{T}^{*}\left(\tau_{T}^{*}\right)}{d \tau_{T}^{* 2}}=0
$$

where $T^{*}=T / T_{p}$ is the temperature relative to that of the plate.

Integrating (26) from 0 to $\tau^{*}$ gives

$$
\frac{d T^{*}}{d \tau_{T}^{*}}\left(\tau_{T}^{*}\right)-\frac{d T^{*}}{d \tau_{T}^{*}}(0)+\mu q\left[\frac{d \varphi_{T}^{*}}{d \tau_{T}^{*}}\left(\tau_{T}^{*}\right)-\frac{d \varphi_{T}^{*}}{d \tau_{T}^{*}}(0)\right]=0 .
$$

Applying the boundary conditions (23c) and (24b) at the surface, this equation becomes

$$
\frac{d T^{*}}{d \tau_{T}^{*}}\left(\tau_{T}^{*}\right)+\pi q \frac{d \varphi_{T}^{*}}{d \tau_{T}^{*}}\left(\tau_{T}^{*}\right)=2 \pi q \varphi_{T s}^{*},
$$

where the subscript $s$ denotes the value at the surface:

$$
\varphi_{T s}^{*}=\varphi_{T}^{*}(0) .
$$

Integrating again gives

$$
T^{*}\left(\tau_{T}^{*}\right)-T_{s}^{*}+\pi q\left[\varphi_{T}^{*}\left(\tau_{T}^{*}\right)-\varphi_{T s}^{*}\right]=2 \pi q \varphi_{T s}^{*} \tau_{T}^{*},
$$

where

$$
T_{s}^{*}=T^{*}(0) \text {. }
$$

This equation shows that the sum of the reduced temperature and $\pi q$ times the reduced thermal flux increases linearly with optical depth through the powder.

At $\tau_{T}^{*}=\tau_{T p}^{*}, T^{*}\left(\tau_{T p}^{*}\right)=T_{p}^{*}=1$. The boundary condition on $\varphi_{T}^{*}$ comes from requiring that the upward radiance at the plate is the sum of radiance emitted by the plate plus the downward radiance from the powder reflected by the plate; that is,

$$
I_{T 1}\left(\tau_{T p}\right)=\varepsilon_{p} \sigma_{0} T_{p}^{4} / \pi+S_{p} I_{T 2}\left(\tau_{T p}\right),
$$

where $S_{p}$ is the reflection coefficient of the plate integrated over the upward hemisphere. By Kirchhoff's law, $S_{p}=1-\varepsilon_{p}$. Converting to reduced quantities, and using (14) and (23b), this boundary condition becomes

$$
\varphi_{T}^{*}\left(\tau_{T p}^{*}\right)+\Delta \varphi_{T}^{*}\left(\tau_{T p}^{*}\right)=\varepsilon_{p}+\left(1-\varepsilon_{p}\right)\left[\varphi_{T}^{*}\left(\tau_{T p}^{*}\right)-\Delta \varphi_{T}^{*}\left(\tau_{T p}^{*}\right)\right],
$$

or

$$
\frac{d \varphi_{T}^{*}}{d \tau_{T}^{*}}\left(\tau_{T p}^{*}\right)=\frac{\varepsilon_{p}}{2-\varepsilon_{p}}\left[1-\varphi_{T_{p}}\left(\tau_{T p}^{*}\right)\right]
$$

However, if the optical thickness of the powder is so large that near the plate the medium is in thermodynamic equilibrium, then $\varphi_{T}^{*} \simeq T_{p}^{* 4}=1$, so that at $\tau_{T}^{*}=\tau_{T p}^{*}$, equation (28) becomes,

$$
1-T_{s}^{*}+\pi q\left(1-\varphi_{T s}^{*}\right)=2 \pi q \varphi_{T s}^{*} \tau_{T p}^{*}
$$

which gives the simpler condition

$$
\varphi_{T s}^{*}=\frac{1-T_{s}+\pi q}{\pi q\left(1+2 \tau_{T p}^{*}\right)} .
$$

In order to complete the solution another relation between $T^{*}$ and $\varphi_{T}^{*}$ is needed. This is provided by the solution to the thermal radiation equation (23a). Unfortunately, this equation is nonlinear and must be solved numerically. A program was written to solve (23) and (28) using the method of finite differences. The program starts by estimating a value for $T_{s}^{*}$ and using (30) to calculate a corresponding value for $\varphi_{T s}^{*}$. Using the boundary conditions (23c) and (24b), the program integrates (23a) and (28) to find values of $\varphi_{T}^{*}$ and $T^{*}$ for increasing values of $\tau_{T}^{*}$. If the guess for $T_{s}^{*}$ is incorrect, $\varphi_{T}^{*}$ and $T^{* 4}$ rapidly diverge. Then a new value for $T_{s}^{*}$ is chosen, and the process repeated until $T^{* 4}$ and $\varphi_{T}^{*}$ converge and also are insignificantly different from 1.00 at $\tau_{T}^{*}=\tau_{T p}^{*}$.

Figure 2 shows the results of such a calculation for $\tau_{T p}^{*}=$ $10, q=0.26$, and $w_{T}^{*}=0.36$. This value for $w_{T}^{*}$ is representative of the single scattering albedo of a particle larger than the wavelength in the thermal IR where strong reststrahlen bands occur, and $q$ is appropriate for an Earth laboratory simulating lunar surface conditions. The reduced temperature $T^{*}$ starts at $T_{s}^{*} \simeq 0.6$ with zero slope, as required by the boundary condition, and then rises with a steadily deceasing slope to 1 at $\tau_{T_{p}}^{*}$. The reduced thermal flux increases 


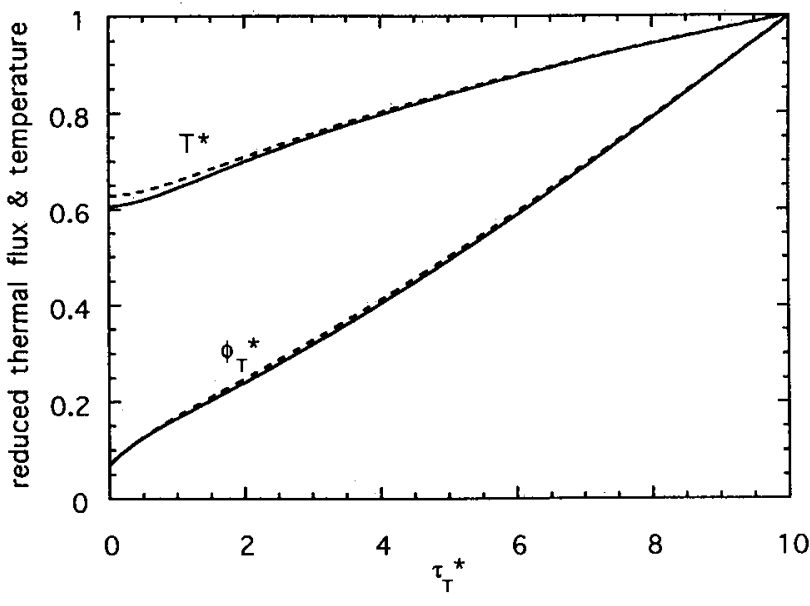

Figure 2. Reduced temperature and thermal radiance as a function of reduced optical depth in a layer of powder in vacuum lying on a heated plate and radiating into a cold environment from its upper surface. Parameters are $\tau_{T y}^{*}=10$, $w_{T}^{*}=0.36, q=0.26$. Solid line is exact calculation; dashed line is boundary layer approximation.

rapidly from its surface value $\varphi_{T s}^{*} \simeq 0.07$ with the required initial slope of $2 \varphi_{T s}^{*}$ and then increases more gradually to 1 at $\tau_{T p}^{*}$. When $2 \gamma_{T}^{*} \tau_{T}^{*}>3, \varphi_{T}^{*}$ is within a few percent of $T^{* 4}$, and the medium is in approximate thermodynamic equilibrium with the thermal radiation.

The rise in temperature near the surface has been pointed out previously by Henderson and Jakosky [1994]. It is caused by the leakage of thermal radiation from the surface, which is also responsible for the near-surface gradient of $\varphi_{T}^{*}$. Within this boundary layer, which is of the order of $1 / \gamma_{T}^{*}$ in optical thickness, the heat carried toward the surface by conduction is converted entirely into thermal radiation.

\subsection{Boundary Layer Approximation}

It would be convenient if an analytical solution to (23a) could be found. This is difficult because the equation is nonlinear. However, an approximate analytical solution that is accurate to a few percent can be derived as follows.

Rewriting (23a),

$$
\vartheta_{T}^{*}=T^{* 4}-\frac{4}{\gamma_{T}^{* 4}} \frac{\partial^{2} \vartheta_{T}^{*}}{\partial \tau_{T^{*}}^{2}}
$$

Now, the deep interior is in thermodynamic equilibrium so that $\varphi_{T}^{*} \simeq T^{* 4}$ there, showing that the second term on the righthand side of the last equation is appreciable only near the surface of the medium. However, at the surface, $d T^{*} / d \tau_{T}^{*}=$ 0 , so that the temperature is almost constant there, $T^{*}=T_{s}^{*}$. In the near-surface region the solution to (23a) with $T^{*}$ constant and that satisfies the boundary condition (23c) is

$$
\varphi_{T}^{*}\left(\tau_{T}^{*}\right)=T_{s}^{* 4}-\frac{\varphi_{T s}^{*}}{\gamma_{T}^{*}} e^{-2 \gamma * T_{T}^{*}} .
$$

These arguments suggest that the thermal flux may be approximated by

$$
\varphi_{T}^{*}\left(\tau_{T}^{*}\right) \simeq T^{* 4}\left(\tau_{T}^{*}\right)-\frac{\varphi_{T s}^{*}}{\gamma_{T}^{*}} e^{-2 \gamma \tau_{T}^{*}}
$$

At $\tau_{T}^{*}=0$, this equation is

$$
\varphi_{T s}^{*}=\frac{\gamma_{T}^{*}}{1+\gamma_{T}^{*}} T_{s}^{* 4}
$$

which is a second relation between $\varphi_{T s}$ and $T_{s}^{*}$ and can be used along with (30) to solve for these quantities.

Using the boundary layer approximation, (28) becomes

$$
\begin{gathered}
T^{*}\left(\tau_{T}^{*}\right)-T_{s}^{*}+\pi q\left[T^{* 4}\left(\tau_{T}^{*}\right)-\frac{\varphi_{T s}^{*}}{\gamma_{T}^{*}} e^{-2 \gamma_{T T}^{*}}-\varphi_{T s}^{*}\right] \\
=2 \pi q \varphi_{T s}^{*} \tau_{T}^{*}
\end{gathered}
$$

At $\tau_{T}^{*}=\tau_{T \rho}^{*}, T^{*}=1$, and this is

$$
\begin{aligned}
1- & {\left[\frac{1+\gamma_{T}^{*}}{\gamma_{T}^{*}} \varphi_{T s}^{*}\right]^{1 / 4}+\pi q\left[1-\frac{\varphi_{T s}^{*}}{\gamma_{T}^{*}} e^{-2 \gamma_{T}^{*} \tau_{p}^{*}}-\varphi_{r_{s}}^{*}\right] } \\
& =2 \pi q \varphi_{T s}^{*} \tau_{T p}^{*},
\end{aligned}
$$

which is a transcendental equation that may be rapidly solved for $\varphi_{T s}^{*}$ by iteration. The solution is then completed by inserting $\varphi_{T s}^{*}$ into (32) and solving for $T_{s}^{*}$. These approximate solutions (equations (31) and (33)) are plotted as the dashed lines in Figure 2.

Converting (33) to real units gives the approximate solution for the temperature

$$
T=T_{s}+\frac{\pi q}{\frac{\sigma_{0}}{\pi} T_{r}^{4}}\left[2 \varphi_{T s} \zeta_{T}^{2} \tau_{T}-\frac{\sigma_{0}}{\pi} T^{4}+\frac{\zeta_{T}}{\gamma_{T}} \varphi_{T s} e^{-2 \zeta r \gamma r \tau T}+\varphi_{T s}\right],
$$

where $T_{s}=T_{r} T_{s}^{*}$ and $\varphi_{T s}=\left(\sigma_{0} / \pi\right) T_{r}^{4} \varphi_{T s}^{*}$

The flux of heat carried by radiative conductivity can be seen explicitly as follows. Substituting the boundary layer approximation for $\varphi_{T}^{*}$ into (27) for the gradient of $T^{*}$ gives

$$
\begin{aligned}
& \frac{d T^{*}}{d \tau_{T}^{*}}\left(\tau_{T}^{*}\right)+\pi q\left[4 T^{* 3}\left(\tau_{T}^{*}\right) \frac{d T^{*}}{d \tau_{T}^{*}}\left(\tau_{T}^{*}\right)+2 \varphi_{T S}^{*} e^{-2 \gamma / \tau T}\right] \\
& \quad=2 \pi q \varphi_{T s^{*}}^{*}
\end{aligned}
$$

Converting to unstarred quantities, this equation can be put into the form

$$
\begin{aligned}
& -k \frac{d T(z)}{d z}+\left[-\frac{4 \sigma_{0} T^{3}(z)}{E_{T}} \frac{d T(z)}{d z}+2 \pi \zeta_{T}^{2} \varphi_{T s} e^{-2 \zeta r \gamma r \tau}\right] \\
& \quad=2 \pi \zeta_{T}^{2} \varphi_{T s} .
\end{aligned}
$$

The first term on the left-hand side of the last equation is the flux of heat carried from the hot plate to the surface by conductivity, while the term in brackets describes the flux of heat carried by radiation. This expression shows that far from the surface, the radiation contribution to the thermal conductivity is proportional to $T^{3}$, as is commonly assumed. However, this assumption is not valid in the boundary layer because it neglects the second term in brackets, which is important near the surface. The IR radiance that is measured by instruments above the surface comes from this boundary layer.

The thermal flux radiated from the surface and the effective temperature at which the surface radiates into the space can be estimated using the boundary layer approximation and equations (14) and (32). The thermal power emitted from unit area of the surface is 


$$
\begin{aligned}
P_{T} & =\int_{2 \pi} I_{T 1}(0) \cos \mathrm{e} d \Omega=\int_{0}^{\pi / 2} I_{T 1}(0) \cos \mathrm{e} 2 \pi \sin e d e \\
& =\pi I_{T 1}(0)=\pi\left[\varphi_{T}(0)+\Delta \varphi_{T}(0)\right]=2 \pi \varphi_{T}(0) \\
& =2 \pi \frac{\sigma_{0}}{\pi} T_{r}^{4} \varphi_{T_{s}}^{*}=2 \sigma_{0} T_{r}^{4} \frac{\gamma_{T}^{*}}{1+\gamma_{T}^{* *}} T_{s}^{* 4}=\frac{2 \gamma_{T}^{*}}{1+\gamma_{T}^{*}} \sigma_{0} T_{s}^{4} \\
& =\varepsilon_{h} \sigma_{0} T_{s}^{4},
\end{aligned}
$$

where $\varepsilon_{h}$ is the hemispherical emissivity. Hence the medium radiates into space at an effective temperature $T_{s}$, which is approximately equal to its actual surface temperature, and with a hemispherical emissivity

$$
\varepsilon_{h}=2 \gamma_{T}^{*} /\left(1+\gamma_{T}^{*}\right)=2 \gamma_{T} /\left(\zeta_{T}+\gamma_{T}\right) .
$$

\section{Planetary Regolith in Equilibrium With Sunlight}

\subsection{Solution}

The second case to be considered is a semi-infinite particulate medium permanently facing the Sun. In the classical steady state problem all absorption and emission of radiation is assumed to take place at the surface, so that the heat equation is simply $\partial^{2} T(z) / \partial z^{2}=0$. The only solution to this problem is $T(z)=$ const. If the temperature of the medium is $T(z)=$ $T_{e}$, and letting the medium have hemispherical albedo $A_{h}$ and hemispherical emissivity $\varepsilon_{h}$, where both $A_{h}$ and $\varepsilon_{h}$ are assumed to be constants of the surface independent of $i$ and $e$, then the visible sunlight absorbed, $\left(1-A_{h}\right) J_{v} \mu_{0}$, is equal to the thermal power radiated, $\varepsilon_{h} \sigma_{0} T_{c}^{4}$, giving

$$
T_{c}=\left[\left(1-A_{h}\right) J_{v} \mu_{0} / \varepsilon_{l l} \sigma_{0}\right]^{1 / 4} .
$$

A more accurate model retains the assumption that the temperature throughout the medium is constant, but solves the visible and IR equations of radiative transfer to obtain $A_{h}$ and $\varepsilon_{h}$. This is done by Hapke [1993a, b], where it is shown that a medium of isotropic scatterers has a hemispherical albedo (directional-hemispherical reflectance)

$$
A_{h}\left(w_{w}, \mu_{0}\right)=1-\gamma_{v} H\left(w_{v}, \mu_{0}\right)
$$

and a hemispherical emissivity

$$
\varepsilon_{h}\left(w_{T}\right)=2 \gamma_{T} H_{1}\left(w_{T}\right),
$$

where $H(w, x)$ is the solution of the integral equation

$$
H(w, x)=1+\frac{w}{2} x H(w, x) \int_{0}^{1} \frac{H\left(w, x^{\prime}\right)}{x+x^{\prime}} d x^{\prime}
$$

and may be approximated by

$$
H(w, x) \simeq(1+2 x) /(1+2 \gamma x),
$$

so that

$$
A_{h}\left(w_{i}, \mu_{0}\right) \simeq\left(1-\gamma_{v}\right) /\left(1+2 \gamma_{v} \mu_{0}\right),
$$

and $H_{1}(w)$ is the first moment of the $H$ function and may be approximated by

$$
H_{1}(w)=\int_{0}^{1} H(w, x) x d x \simeq \frac{1}{1+\gamma}\left(1+\frac{1}{6} \frac{1-\gamma}{1+\gamma}\right),
$$

giving

$$
\varepsilon_{l l}\left(w_{T}\right) \simeq \frac{2 \gamma_{T}}{1+\gamma_{T}}\left(1+\frac{1}{6} \frac{1-\gamma_{T}}{1+\gamma_{T}}\right)
$$

Thus,

$$
\begin{aligned}
T_{c} & =\left[\frac{J_{v} \mu_{0}}{\sigma_{0}} \frac{\gamma_{v} H\left(w_{v}, \mu_{0}\right)}{2 \gamma_{T} H_{1}\left(w_{T}\right)}\right]^{1 / 4} \\
& \simeq\left[\frac{J_{v} \mu_{0}}{\sigma_{0}} \gamma_{v} \frac{1+2 \mu_{0}}{1+2 \gamma_{v} \mu_{0}} \frac{1+\gamma_{T}}{2 \gamma_{T}} \frac{1}{1+\frac{1}{6} \frac{1-\gamma_{T}}{1+\gamma_{T}}}\right]^{1 / 4} .
\end{aligned}
$$

If the IR reflectance of the medium is small, as is often the case because of the strong reststrahlen bands there, $\gamma_{T}$ is close to one and the factor $\left(1-\gamma_{T}\right) / 6\left(1+\gamma_{T}\right)$ in (44) and (45) may be neglected.

The restrictions that the temperature of the medium is constant and that the particles scatter isotropically will now be dropped. Instead, the steady state heat equation and the visible and IR radiative transfer equations given in section 2 will be solved.

For the steady state, $\partial T^{*} / \partial t^{*}=0, \mu_{0 z^{* *}}=$ const and $F\left(t^{*}\right)=1$. Then (22)-(24) are

$$
\begin{gathered}
\frac{1}{4} \frac{d^{2} \varphi_{v}^{*}}{d \tau_{v}^{* 2}}=\gamma_{v}^{* 2} \varphi_{v}^{*}-\frac{w_{v}^{*}}{4 \pi} \frac{1+\beta_{v} / 2 \mu_{0 v}^{*}}{1-\beta_{v}} e^{-\tau_{v}^{*} / \mu_{v i v}^{*}}, \\
\varphi_{v}(0)-\frac{1}{2} \frac{d \varphi_{v}^{*}}{d \tau_{v}^{*}}(0)-\frac{w_{v}^{*}}{4 \pi} \frac{\beta_{v}}{1-\beta_{v}}, \\
\frac{1}{4} \frac{d^{2} \varphi_{T}^{*}}{d \tau_{T}^{* 2}}=\gamma_{T}^{* 2}\left(\varphi_{T}^{*}-T^{* 4}\right), \\
\varphi_{T}^{*}(0)=\frac{1}{2} \frac{d \varphi_{T}^{*}}{d \tau_{T}^{*}}(0), \\
\frac{d^{2} T^{*}}{d \tau_{T}^{* 2}}+\pi q \frac{d^{2} \varphi_{T}^{*}}{d \tau_{T}^{* 2}}=-R q \gamma_{v}^{* 2}\left[e^{-\pi_{v}^{*} / \mu_{i n}^{*}}+4 \pi \varphi_{v}^{*}\right], \\
\frac{d T^{*}}{d \tau_{T}^{*}}(0)=0 .
\end{gathered}
$$

Equation (46a) for the visible flux is independent of the other two equations and can be solved separately. The solution satisfying the boundary condition that the flux is finite at infinity is

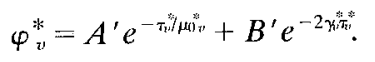

The coefficient $B^{\prime}$ can be found by substituting this solution into the differential equation (46a) and equating coefficients of $\exp \left(-\tau_{v}^{*} / \mu_{0 v}^{*}\right) ; A^{\prime}$ can be found by substituting the solution into the boundary condition (46b). This gives

$$
\begin{gathered}
A^{\prime}=-\frac{w_{v}^{*}}{4 \pi} \frac{4 \mu_{0 v}^{* 2}\left(1+\beta_{v} / 2 \mu_{0 v}^{*}\right)}{\left(1-\beta_{v}\right)\left(1-4 \gamma_{v}^{* *} \mu_{0 v}^{* 2}\right.} \\
B^{\prime}=\frac{1}{4 \pi} \frac{2 \mu_{0 v}^{*}\left(1-\gamma_{v}^{*}\right)\left[\left(1+2 \mu_{0 v}^{*}\right)+\beta_{v}\left(1+2 \gamma_{v}^{* 2} \mu_{0 v}^{*}\right)\right]}{\left(1-4 \gamma_{v}^{* 2} \mu_{0 v}^{* 2}\right)\left(1-\beta_{v}\right)} .
\end{gathered}
$$

Inserting this solution into (48a), the heat transfer equation becomes 


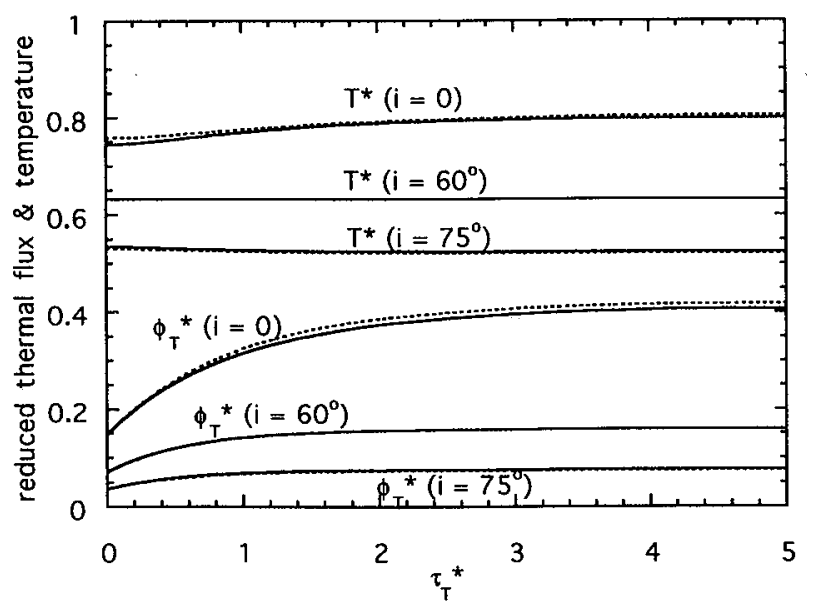

Figure 3. Reduced temperature and thermal radiance as a function of reduced optical depth in a regolith in vacuum in equilibrium with sunlight, showing the effect of varying the angle of incidence $i$. Parameters are $w_{v}^{*}=0.36, w_{T}^{*}=0.36$, $q=0.26$. Solid line is exact calculation; dashed line is boundary layer approximation.

$$
\frac{d^{2} T^{*}}{d \tau_{T^{*}}^{2}}+\mu q \frac{d^{2} \varphi_{T}^{*}}{d \tau_{T}^{* 2}}=-R q \gamma_{v}^{* 2}\left[A e^{-R \tau^{*} / \mu \mu_{v}^{*} v}+B e^{-2 \gamma_{v}^{*} R \tau T}\right]
$$

where

$$
\begin{gathered}
A=1+4 \pi A^{\prime} \\
=\frac{1+2 \mu_{0 v}^{* 2}}{1-4 \gamma_{v}^{* 2} \mu_{0 v}^{* 2}} \frac{\left(1-2 \mu_{0 v}^{*}-\beta_{v}\left(1-2 \gamma_{v}^{* 2} \mu_{0 v}^{*}\right)\right.}{1-\beta_{v}} \\
B=4 \pi B^{\prime}=\frac{2 \mu_{0_{v}}^{*}\left(1-\gamma_{v}^{*}\right)\left[\left(1+2 \mu_{0 v}^{*}\right)+\beta_{v}\left(1+2 \gamma_{v}^{* 2} \mu_{0 v}^{*}\right)\right]}{\left(1-4 \gamma_{v}^{* 2} \mu_{0 v}^{* 2}\right)\left(1-\beta_{v}\right)} .
\end{gathered}
$$

Integrating (50a) once with respect to $\tau_{T}^{*}$ from 0 to $\tau_{T}^{*}$ and applying the boundary conditions gives

$$
\begin{aligned}
& \frac{d T^{*}}{d \tau_{T}^{*}}\left(\tau_{T}^{*}\right)+\pi q\left[\frac{d \varphi_{T}^{*}}{d \tau_{T}^{*}}\left(\tau_{T}^{*}\right)-2 \varphi_{T S}^{*}\right] \\
& \quad=q \gamma_{v}^{* 2}\left[A \mu_{0 v}^{*}\left(e^{-R \tau_{i}^{*} / \mu_{0 *}^{*}}-1\right)+\frac{B}{2 \gamma_{v}^{*}}\left(e^{-2 \gamma_{*}^{*} R \tau^{*}}-1\right)\right],
\end{aligned}
$$

where

$$
\varphi_{T s}^{*}=\varphi_{T}^{*}(0)
$$

Integrating again gives

$$
\begin{aligned}
T^{*}\left(\tau_{T}^{*}\right) & -T_{s}^{*}+\pi g\left[\varphi_{T}^{*}\left(\tau_{T}^{*}\right)-\varphi_{T s}^{*}-2 \varphi_{T s}^{*} \tau_{T}^{*}\right] \\
= & -q \gamma_{v}^{* 2}\left\{A \mu_{0 v}^{*}\left[\frac{\mu_{0} v^{*}}{R}\left(e^{-R \tau_{T}^{*} / \mu_{l, v}^{*}}-1\right)+\tau_{T}^{*}\right]\right. \\
& \left.+\frac{B}{2 \gamma_{v}^{*}}\left[\frac{1}{2 \gamma_{v}^{* R}}\left(e^{-2 \gamma_{r}^{*} R t T_{T}^{*}}-1\right)+\tau_{T}^{*}\right]\right\},
\end{aligned}
$$

where

$$
T_{s}^{*}=T^{*}(0)
$$

However, $T^{*}$ must retain finite as $\tau_{T}^{*} \rightarrow \infty$. The only way this can be true is if the sum of the coefficients of $\tau_{T}^{*}$ vanishes; thus

$$
2 \pi \varphi_{T s}^{*}=\gamma_{v}^{* 2}\left(A \mu_{0 v}^{*}+B / 2 \gamma_{v}^{*}\right)
$$

After substituting (50b) and (50c) and simplifying algebraically, this becomes

$$
\begin{aligned}
\varphi_{T s}^{*}= & \frac{\gamma_{v}^{*} \mu_{0 v}^{*}}{2 \pi\left(1+2 \gamma_{v}^{*} \mu_{0 v}^{*}\right)} \\
& \cdot \frac{\left(1+2 \mu_{0 v}^{*}\right)+\beta_{v}\left(1-2 \gamma_{v}^{*}-2 \gamma_{v}^{* 2} \mu_{0 v}^{*}\right)}{\left(1-\beta_{v}\right)} .
\end{aligned}
$$

Thus the solution to the heat transfer equation is

$T^{*}\left(\tau_{T}^{*}\right)-T_{s}^{*}+\pi q\left(\varphi_{T}^{*}\left(\tau_{T}^{*}\right)-\varphi_{T s}^{*}\right]$

$=\frac{q}{R}\left[A \gamma_{v}^{* 2} \mu_{0 v}^{* 2}\left(1-e^{-R \tau \tau^{*} / / \mu \nu_{v}^{*}}\right)+B / 4\left(1-e^{-2 \gamma_{i}^{*} R \tau \tau^{*}}\right)\right]$.

Now, deep in the interior of the medium as $\tau_{T}^{*} \rightarrow \infty, \varphi_{\nu}^{*} \rightarrow$ $0, T^{*}$ and $\varphi_{T}^{*}$ become constant; hence $d^{2} \varphi_{T}^{*} / d \tau_{T}^{2}=0$, and $\varphi_{T}^{*}(\infty)=T^{* 4}(\infty)$. Thus, as $\tau \rightarrow \infty$, equation (57) becomes

$$
\begin{aligned}
T^{*}(\infty) & -T_{s}^{*}+\pi q T^{* 4}(\infty) \\
= & q\left[\pi \varphi_{T s}^{*}+(1 / R)\left(\gamma_{v}^{* 2} \mu_{v_{v}}^{* 2} A+B / 4\right)\right] .
\end{aligned}
$$

This is an alternate boundary condition.

Equations (47) and (48) were solved numerically by the method of finite differences in a manner similar to that used in section 3 . The procedure starts by guessing a value for $T_{s}^{*}$ and integrating inward from the surface, with $\varphi_{T s}^{*}$ given by (56). If the guess for $T_{s}^{*}$ is incorrect, the numerical solutions for $\varphi_{T}^{*}$ and $T^{* 4}$ rapidly diverge, in which case the integration is stopped, a new value for $T_{s}^{*}$ is estimated, and the procedure is repeated until a value is found for $T_{s}^{*}$ that allows $\varphi_{r}^{*}$ and $T^{* 4}$ to converge with increasing $\tau_{T}^{*}$.

The results of the numerical calculation are shown as the solid lines in Figures 3-6, which illustrate the effects of varying the parameters of the solutions. In Figures 3-6 it was assumed that the particles are isotropic scatterers and that $E_{z}=E_{T}$, so

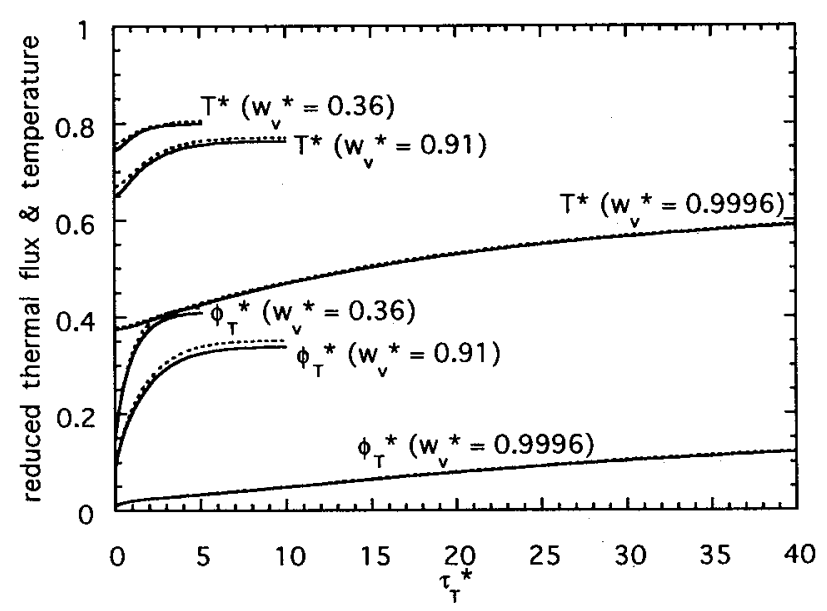

Figure 4. Reduced temperature and thermal radiance as a function of reduced optical depth in a regolith in vacuum in equilibrium with sunlight, showing the effect of varying the visible single scattering albedo $w_{\eta}^{*}$. Parameters are $w_{T}^{*}=$ $0.36, q=0.26, i=0$. Solid line is exact calculation; dashed line is boundary layer approximation. 
that $R=1$ and $\tau_{T}^{*}=\tau_{v}^{*}$. The value for the visual single scattering albedo of $w_{v}^{*}=0.36$ might be representative of a relatively low albedo body, such as the Moon or an asteroid. The high value, $w_{v}^{*}=0.91$, might be representative of somewhat dirty ice on the surface of a rocky-icy satellite, and $w_{v}^{*}=$ 0.9996 , representative of clean, fresh frost or snow. In the thermal IR, $w_{T}^{*}=0.36$, is probably representative of most materials. A value of $w_{T}^{*}=0.91$ probably is unrealistically high for most materials of planetary interest because reststrahlen bands are common in most materials at IR wavelengths; however, it is included to show the effect of a material of high IR albedo, such as an alkali halide.

In all cases, as the optical depth $\tau_{T}^{*}$ increases, $\varphi_{T}^{*}$ rises rapidly from a value of $\varphi_{T s}^{*}$ at the surface, as required by the boundary condition, and then levels off either to a constant value or to a more gentle rate of increase. The rapid rise is a direct result of leakage of thermal radiation from the surface. The medium approaches thermodynamic equilibrium when $\tau_{T}^{*}>3$.

The behavior of $T^{*}$ is more complex. The slope of $T^{*}$ is zero at the surface, as required by the boundary condition, but as $\tau_{T}^{*}$ increases, $T^{*}$ may exhibit a positive or negative slope, depending on the relative value of $\gamma_{T}^{*}$ compared with $\gamma_{v}^{*}$ and $\mu_{0}^{*}$. If these quantities are comparable, $T^{*}$ has a positive slope. However, the slope of $T^{*}$ may be small or even negative if $\gamma_{T}^{*}$ is small. Heating by unscattered visible radiation occurs over an optical depth of the order of $1 / \mu_{0 v}^{*}=1 / \zeta_{v}^{2} \mu_{0}$ and by multiply scattered visible radiation over $1 / \gamma_{v}^{*}=\zeta_{v} / \gamma_{v}$, but the IR flux is radiated from the surface over an optical depth of the order of $1 / \gamma_{T}^{*}=\zeta_{T} / \gamma_{T}$. If $1 / \gamma_{T}^{*}$ is larger than $1 / \mu_{0 y}^{*}$, or $1 / \gamma_{v}^{*}$, then heat must be conducted from the visible heating source, which is closer to the surface, to supply power to the deeper IR radiative sink. This requires a negative temperature gradient. Thus, when $i$ is large a negative greenhouse effect can occur. Henderson and Jakosky [1994, also submitted manuscript, 1995] have previously pointed out that large positive subsurface temperature gradients are possible, but they did not investigate cases where the gradient was small or negative. It should also be emphasized that the widths of the gradients in actual depth

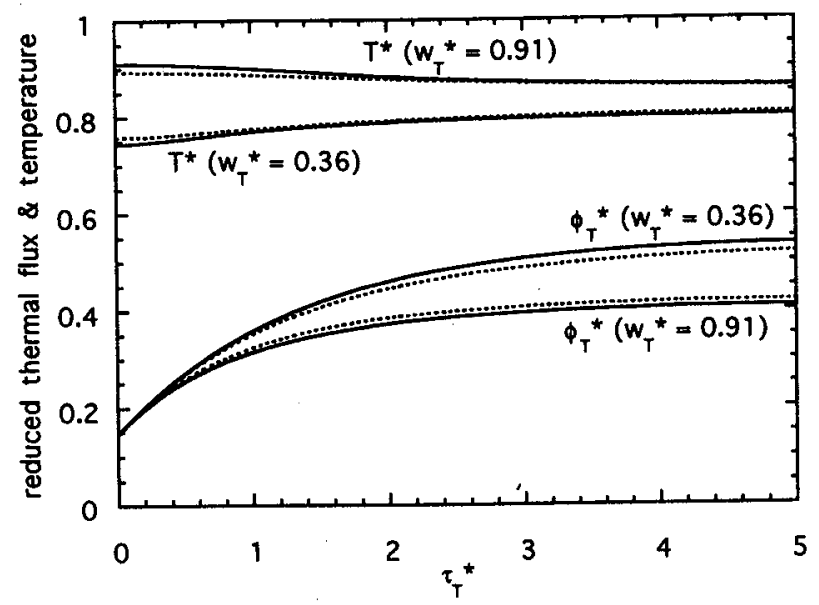

Figure 5. Reduced temperature and thermal radiance as a function of reduced optical depth in a regolith in vacuum in equilibrium with sunlight, showing the effect of varying the thermal single scattering albedo $w_{T}^{*}$. Parameters are $w_{v}^{*}=$ $0.36, q=0.26, i=0$. Solid line is exact calculation; dashed line is boundary layer approximation.

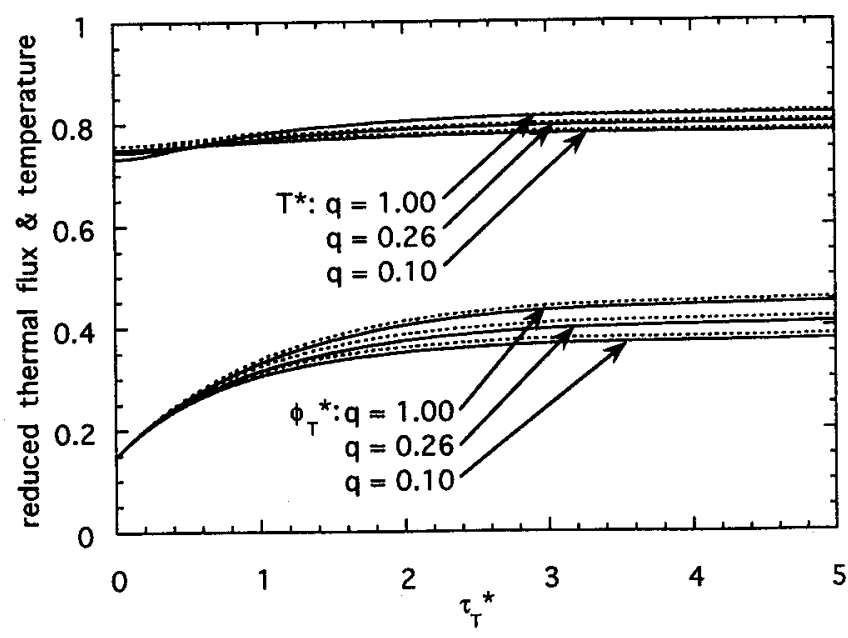

Figure 6. Reduced temperature and thermal radiance as a function of reduced optical depth in a regolith in vacuum in equilibrium with sunlight, showing the effect of varying the radiative conductivity parameter $q$. Parameters are $w_{\nu}^{*}=$ $0.36, w_{T}^{*}=0.36, i=0$. Solid line is exact calculation; dashed line is boundary layer approximation.

$z$ (rather than in reduced depth $\tau_{T}^{*}$ ) will depend inversely on the particle density or filling factor.

As shown in Figure 6, the parameter $q$ has a relatively small effect on the reduced variables (although, of course, the absolute effect can be large). The reason can be seen from (58) for the total rise in temperature. As $q$ increases, the visible heating increases, which increases $T^{*}(\infty)-T_{s}^{*}$, but the radiative conductivity also increases, which limits the rise in temperature.

Substituting the boundary layer approximation

$$
\varphi_{T}^{*}\left(\tau_{T}^{*}\right)-T^{* 4}\left(\tau_{T}^{*}\right)-\frac{\varphi_{T s}^{*}}{\gamma_{T}^{*}} e^{-2 \gamma_{T}^{*} T^{*}},
$$

into (57) gives an approximate expression for $T^{*}$,

$$
\begin{aligned}
T^{*}\left(\tau_{T}^{*}\right) & \left.=T_{s}^{*}-\pi q T^{* 4}\left(\tau_{T}^{*}\right)-\frac{1}{\gamma_{T}^{*}} \varphi_{T s}^{*} e^{-2 \gamma_{T}^{*} \pi^{*}}-\varphi_{T s}^{*}\right] \\
+ & \frac{q}{R}\left[\gamma_{\nu}^{* 2} \mu_{0 v}^{2} A\left(1-e^{-R \tau_{T}^{*} / \mu_{\left(\nu^{\prime}\right.}^{*}}\right)+\frac{B}{4}\left(1-e^{-2 \gamma_{r}^{*} R \tau T}\right)\right],
\end{aligned}
$$

where

$$
T_{s}^{*}=\left(\frac{1+\gamma_{T}^{*}}{\gamma_{T}^{*}} \varphi_{T s}^{*}\right)^{1 / 4},
$$

and $\varphi_{T s}^{*}$ is given by (56). The boundary layer approximation (equations (59)-(61) is plotted as dashed lines in Figures 3-6. The exact and approximate solutions are within a few percent of one another everywhere.

\subsection{Emitted Thermal Radiance and Hemispherical Albedo}

The thermal flux radiated from the surface, the effective temperature at which the surface radiates into space, and the hemispherical emissivity can be determined in the same way as was done in section 3 for the case of the powder heated from below. From (36), the thermal power emitted from the surface is 


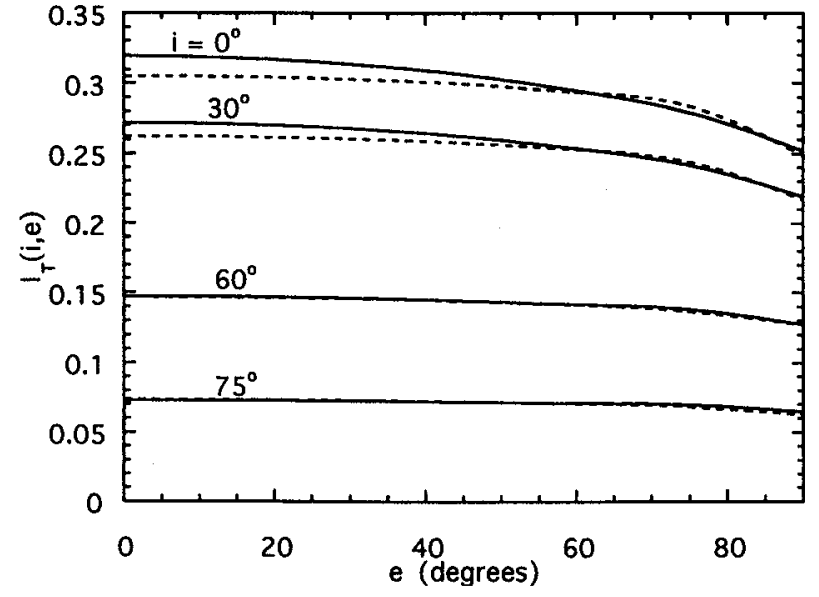

Figure 7. Thermal flux emerging from the surface of a regolith in vacuum in equilibrium with sunlight as a function of the angle of emergence $e$ for various angles of incidence $i$. Parameters are $w_{v}=0.36, w_{T}=0.36, \beta_{v}=0, \beta_{T}=0, q=0.26$. Solid line is exact calculation; dashed line is constant subsurface temperature.

$$
\begin{aligned}
P_{T} & =\int_{2 \pi} I_{T 1}(0) \cos e d \Omega=2 \pi \varphi_{T}(0)=2 \pi J_{\nu} \varphi_{T s}^{*} \\
& =2 \pi \frac{\sigma_{0}}{\pi} T_{r}^{4} \frac{\gamma_{T}^{*}}{1+\gamma_{T}^{*}} T_{s}^{* 4}=\frac{2 \gamma_{T}^{*}}{1+\gamma_{T}^{*}} \sigma_{0} T_{s}^{4} .
\end{aligned}
$$

Hence, to the accuracy of the boundary layer approximation, the effective radiating temperature of the surface is

$$
T_{s}=T_{r} T_{s}^{*}=\left(\frac{\pi}{\sigma_{0}} J_{v} \frac{1+\gamma_{T}^{*}}{\gamma_{T}^{*}} \varphi_{T s}^{*}\right)^{1 / 4},
$$

and the hemispherical emissivity is

$$
\varepsilon_{h}=2 \gamma_{T}^{*}\left(1+\gamma_{T}^{*}\right)=2 \gamma_{T} /\left(\zeta_{T}+\gamma_{T}\right),
$$

which is the same result obtained in section 3.2 for the powder heated by a bottom plate.

The visible hemispherical albedo (directional-hemispherical reflectance) $A_{h}\left(\mu_{0}\right)$ of a surface in radiative equilibrium with sunlight can be found from (56) for $\varphi_{T s}^{*}$ by noting that the thermal power emitted per unit area must equal the visible power absorbed per unit area; that is,

$$
P_{T}=2 \pi J_{v} \varphi_{r_{s}}^{*}=J_{v} \mu_{0}\left[1-A_{h}\left(\mu_{0}\right)\right] .
$$

Thus

$$
\begin{aligned}
A_{h}\left(\mu_{0}\right)= & 1-\frac{2 \pi}{\mu_{0}} \varphi_{T s}^{*}=1-\frac{\zeta_{v}^{2} \gamma_{v}^{*}}{\left(1+2 \gamma_{v}^{*} \mu_{0 v}^{*}\right)} \\
& \cdot \frac{\left(1+2 \mu_{0 v}^{*}\right)+\beta_{v}\left(1-2 \gamma_{v}^{*}-2 \gamma_{v}^{* 2} \mu_{0 v}^{*}\right)}{1-\beta_{v}} \\
= & 1-\frac{\zeta_{v} \gamma_{v}}{1+2 \zeta_{v} \gamma_{u} \mu_{0}} \\
& \cdot \frac{\left(1+2 \zeta_{v}^{2} \mu_{0}\right)+\beta_{v}\left(1-2 \gamma_{v} / \zeta_{v}-2 \gamma_{v}^{2} \mu_{0}\right)}{1-\beta_{v}}
\end{aligned}
$$

which can be further simplified to

$$
A_{h}=\frac{\zeta_{v}-\gamma_{v}}{1+2 \zeta_{v} \gamma_{v} \mu_{0}} \frac{\zeta_{v}-\beta_{v} \gamma_{v}}{1-\beta_{v}}
$$

Comparing (65a) and (38) for $A_{h}$ shows that the two are identical when $\beta_{v}=0$. Comparing (64) and (39) for $\varepsilon_{h}$ shows that when $\beta_{T}=0$, the two are the same if $H_{1}\left(w_{T}\right)$ is replaced by its low-albedo approximation $H_{1}\left(w_{T}\right)=1 /\left(1+\gamma_{T}\right)$. This approximation is probably valid in most cases of interest, because strong reststrahlen bands cause the IR albedos of most substances to be small.

By symmetry [Hapke, 1993a], the visible hemisphericaldirectional reflectance has exactly the same form as $(65 a)$ or (65b), except that $\mu_{0}$ is replaced by $\mu$. Also, the IR directionalhemispherical, and hemispherical-directional reflectances have the same forms as their visible counterparts, except that subscripts $v$ are replaced by $T$.

Hapke [1993a] shows that the IR radiance emerging from the surface of a particulate medium is given by

$$
\begin{aligned}
I_{T}\left(\mu_{0}, \mu\right)= & \int_{0}^{\infty}\left[\frac{w_{T}}{4 \pi} \int_{4 \pi} I_{T}\left(\tau_{T}, \mu, \Omega^{\prime}\right) p_{T}\left(\Omega^{\prime}, \Omega\right) d \Omega^{\prime}\right. \\
& \left.+\gamma_{T}^{2} \frac{\sigma_{0}}{\pi} T^{4}\left(\tau_{T}\right)\right] e^{-\tau / \mu} \frac{d \tau_{T}}{\mu} .
\end{aligned}
$$

In the two-stream approximation with the hemispherical asymmetry factor, the integral over solid angle is

$$
\begin{aligned}
& \frac{w_{T}}{4 \pi} \int_{4 \pi} I_{T}\left(\tau_{T}, \mu, \Omega^{\prime}\right) p_{T}\left(\Omega^{\prime}, \Omega\right) d \Omega^{\prime}=w_{T} \varphi_{T}\left(\tau_{T}\right) \\
& \quad+\beta_{T} w_{T} \Delta \varphi_{T}\left(\tau_{T}\right) .
\end{aligned}
$$

Substituting this into (66), changing to reduced quantities, and using (23b) gives

$$
\begin{gathered}
I_{T}\left(\mu_{0}, \mu\right)=J_{v} \int_{0}^{\infty}\left[\frac{\zeta_{T}^{2}}{1-\beta_{T}} w_{T}^{*} \varphi_{T}^{*}\left(\tau_{T}^{*}\right)+\frac{1}{2} \frac{\zeta_{T}^{2}}{1-\beta_{T}}\right. \\
\left.\cdot \beta_{T} w_{T}^{*} \frac{d \varphi_{T}^{*}\left(\tau_{T}^{*}\right)}{d \tau_{T}^{*}}+\zeta_{T}^{2} \gamma_{T}^{* 2 * 4}\left(\tau_{T}^{*}\right)\right] e^{-\tau_{T}^{*} / \mu \frac{*}{T}} \frac{d \tau_{T}^{*}}{\mu_{T}^{*}} .
\end{gathered}
$$

Equation (68) was evaluated numerically to give $I_{T}^{*}\left(\mu_{0}, \mu\right)$ $=I_{T}\left(\mu_{0}, \mu\right) / J_{v}$ versus $e$ for a lunar-like regolith. The results are shown as the solid lines in Figure 7.

\subsection{Directional Emissivity and the Constant Subsurface Temperature Approximation}

The integrand in (68) contains terms proportional to $\varphi_{T}^{*}$, $d \varphi_{T}^{*} / d \tau_{T}^{*}$ and $T^{* 4}$ multiplied by $\exp \left(-\tau_{T}^{*} / \mu_{T}^{*}\right)$. This integrand is appreciable only over a distance of the order of $1 / \mu_{T}^{*}$ from the surface. However, because of the zero initial slope of $\mathrm{T}^{*}$, within the layer, $T^{*}$ is nearly constant. Hence the boundary layer approximation with $T^{*}=T_{s}^{*}$ may be used to obtain an approximate evaluation of (68). This gives

$$
\begin{aligned}
& I_{T}\left(\mu_{0}, \mu\right) \simeq=J_{y} T_{s}^{* 4} \frac{\zeta_{T}^{2} \gamma_{T}^{*}}{1+2 \gamma_{T}^{*} \mu_{T}^{*}} \\
& \cdot \frac{\left(1+2 \mu_{T}^{*}\right)+\beta_{T}\left(1-2 \gamma_{T}^{*}-2 \gamma_{T}^{*} \mu_{T}^{*}\right)}{1-\beta_{T}}
\end{aligned}
$$

Writing this in the form 


$$
I_{T}\left(\mu_{0}, \mu\right) \simeq \varepsilon_{d}(\mu) \frac{\sigma_{0}}{\pi} T_{s}^{4}
$$

shows that the effective radiating temperature of the medium is approximately

$$
T_{s} \simeq T_{r}\left(\frac{\zeta_{T}+\gamma_{T}}{\gamma_{T}} \varphi_{T s}^{*}\right)^{1 / 4}
$$

where $\varphi_{r s}^{*}$ is given by (56) and the directional emissivity is given by the approximate expression

$$
\varepsilon_{d}(\mu) \simeq \frac{\zeta_{T} \gamma_{T}}{1+2 \zeta_{T} \gamma_{T}^{\mu}} \frac{\left(1+2 \zeta_{T}^{2} \mu\right)+\beta_{T}\left(1-2 \gamma_{T} / \zeta_{T}-2 \gamma_{T}^{2} \mu\right)}{1-\beta_{T}}
$$

If the particles scatter isotropically in the IR $\left(\beta_{T}=0\right)$, the emissivity is

$$
\varepsilon_{d}(\mu)=\gamma_{T} \frac{1+2 \mu}{1+2 \gamma_{T} \mu}=\gamma_{T} H\left(w_{T}, \mu\right)
$$

which has been previously derived by Hapke [1993a].

It should be emphasized that (56) for $\varphi_{T s}^{*}$ and (65a) or (65b) for $A_{h}\left(\mu_{0}\right)$ and the visible and thermal-IR directionalhemispherical and hemispherical-directional reflectances are exact and do not depend on the validity of the boundary layer approximation. Equations (64) for $\varepsilon_{h}$ and (71a) for $T_{s}$ depend on the boundary layer approximation but not the constant subsurface temperature approximation. Expression (71b) for $\varepsilon_{d}(\mu)$ depends on the validity of both the boundary layer and constant subsurface temperature approximation. All of these expressions include the effects of the gradient in $\varphi_{T}^{*}$, which is closer to the surface than the gradient in $T^{*}$.

By combining (56), (71a), (71b), and (72), the surface temperature can be written in the form $T_{s}=\left[\left(1-A_{h}\right) J_{v} \mu_{0}\right.$ / $\left.\varepsilon_{h} \sigma_{0}\right]^{1 / 4}$, which is identical to the expression for the radiative equilibrium temperature $T_{e}$ derived at the beginning of this section under the simple assumption that the temperature of the medium is constant. Thus, allowing for a solid-state greenhouse increases or decreases the temperature distribution in the interior of the medium but does not alter the surface temperature.

The approximate expressions (69)-(71) for $I_{T}\left(\mu_{0}, \mu\right)$ versus $e$ were calculated for the same values of the parameters as used in the numerical evaluation of (68). The results are shown as the dashed lines in Figure 7. The agreement between the exact and approximate values is good when either $i$ or $e$ is large, but there is a discrepancy of as much as $5 \%$ when both $i$ and $e$ are small because of the neglect of the subsurface temperature gradient in evaluating the integral in $I_{T}\left(\mu_{0}, \mu\right)$. If $e$ is large, the integrand in the expression for $I_{T}$ is appreciable only close to the surface, where the temperature is approximately constant. If $i$ is large, the temperature gradient is small and its neglect introduces little error. The gradient influences the emergent radiance only when the surface is both viewed and illuminated at nearly vertical angles.

The hemispherical-directional reflectance in the thermal-IR is given by (65) with $\mu_{0}$ replaced by $\mu$ and subscript $v$ replaced by $T$. By Kirchhoff's law [Hapke, 1993a], the directional emissivity is the complement of the hemispherical-directional reflectance. Comparing (71b) with (65a) shows that this is indeed true. However, (71b) is an approximation which is valid only when a large subsurface temperature gradient is not present. This is in agreement with the result previously obtained exper-

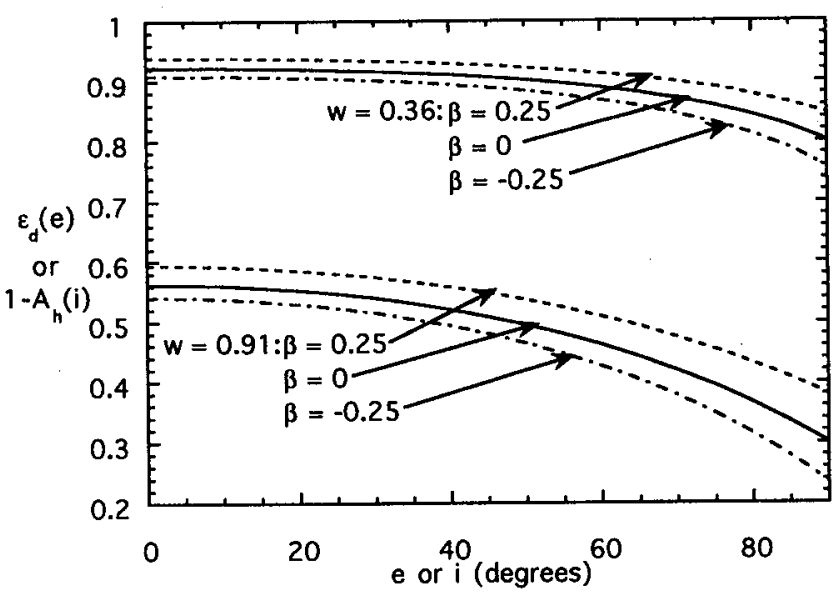

Figure 8. Directional emissivity $\varepsilon_{d}(\mu)$ as a function of angle of emergence $e$ and the complement of the hemispherical albedo $1-A_{h}\left(\mu_{0}\right)$ as a function of angle of incidence $i$ for different values of the thermal or visible single scattering albedo $w$ and hemispherical asymmetry parameter $\beta$.

imentally by Salisbury et al. [1994], who showed that Kirchhoff's law is violated in media with large near-surface temperature gradients.

The effect of single scattering albedo and asymmetry factor on $\varepsilon_{d}(\mu)$ is plotted for a high and a low albedo in Figure 8 . Because $1-A_{h}\left(\mu_{0}\right)$ has the same functional dependence on $\mu_{0}$ as $\varepsilon_{d}(\mu)$ does on $\mu$, these curves also describe 1 $A_{h}\left(\mu_{0}\right)$, as indicated in Figure 8 . Making the particles more forward scattering increases the directional emissivity and decreases the hemispherical albedo but does not change the shapes of the curves appreciably.

\subsection{Spectral Boundary Layer Approximation}

For future use, it is of interest to write down explicitly the unreduced boundary layer approximation for the thermal and spectral fluxes in the IR. Converting the boundary layer approximation (equation (59)) to unstarred quantities gives for the radiative flux integrated over thermal wavelengths,

$$
\varphi_{T}\left(\tau_{T}\right) \simeq \frac{\sigma_{0}}{\pi} T^{4}\left(\tau_{T}\right)-\frac{\zeta_{T}}{\gamma_{T}} \varphi_{r_{s}} e^{-2 \zeta r \gamma \tau \tau_{T}}
$$

By analogy, the boundary layer approximation for the radiance averaged over angle in the thermal-IR wavelength region where the visible source spectral irradiance is negligible is

$$
\varphi_{\lambda}\left(\tau_{\lambda}\right)=\frac{1}{\pi} U_{\lambda}(T)-\frac{\zeta_{\lambda}}{\gamma_{\lambda}} \varphi_{\lambda s} e^{-2 \zeta_{\lambda} \gamma_{\lambda} \tau_{\lambda}},
$$

where

$$
\begin{gathered}
\varphi_{\lambda s}=\frac{\gamma_{\lambda}}{\zeta_{\lambda}+\gamma_{\lambda}} \frac{1}{\pi} U_{\lambda}\left(T_{s}\right), \\
T_{s}=T_{r} T_{s}^{*},
\end{gathered}
$$

$T_{s}^{*}$ is given by (61) and (56), and $T_{r}$ is the blackbody radiative equilibrium temperature.

\section{Conclusions}

The temperature and thermal radiance in a planetary regolith are governed by three equations: the equation of radiative 
transfer for visible and for IR radiation and the heat transfer equation. These equations were written in terms of the twostream approximation, including anisotropic scattering, which allows numerical solutions to be rapidly obtained. An approximate analytic solution, the boundary layer approximation, to the nonlinear thermal radiative transfer equation was obtained. This approximation allows an analytic solution to the heat equation to be derived that gives the distribution of temperature and thermal radiance as a function of depth, and that is accurate to a few percent. The equations intrinsically contain radiative conductivity and result in a changing temperature with depth, i.e., a solid-state greenhouse effect.

Using this formalism, the steady state cases of a powder heated form below in the laboratory and a planetary regolith in equilibrium with sunlight were studied. Subsurface temperature gradients that are positive, negative, or zero can occur, depending on the thermal and radiative constants and the geometry. Large gradients may occur in a layer only a few particles thick or may be spread out over a large depth, depending on the visible and IR radiative constants of the medium. Approximate analytic expressions for the directionalhemispherical albedo (equation (65)), the hemispherical emissivity (equation (64)), and the directional emissivity (equation (71)) for regoliths of anisotropically scattering particles were derived. This model was also used to study three interesting problems in the thermal emission from planetary regoliths by Hapke [this issue]. Using the same formalism, an improved expression for the bidirectional reflectance of a regolith of highly anisotropically scattering particles was also derived and will be the subject of a separate paper (B. Hapke, manuscript in preparation, 1996).

\section{Notation}

$A_{h}$ hemispherical albedo, equal to directional-

hemispherical reflectance.

$C$ specific heat per unit mass.

$c_{0}$ speed of light in vacuum.

$d_{j}$ mean diameter of a particle of type $j$, equal to $\left(4 \sigma_{j} / \pi\right)^{1 / 2}$.

$E_{\lambda}(z), E_{v}(z), E_{T}(z)$ spectral visible, and thermal volume extinction coefficients, equal to

$\sum_{j} N_{j}(z) \sigma_{j} Q_{E \lambda j}, \sum_{j} N_{j}(z) \sigma_{j} Q_{E v j}$, and

$\sum_{j} N_{j}(z) \sigma_{j} Q_{E v T}$, respectively.

$e$ zenith angle at which radiance emerges from the medium toward the detector.

$\mathscr{E}_{\lambda}, \mathscr{E}_{T}$ spectral and thermal volume emission coefficients, equal to $K_{\lambda}(z) / E_{\lambda}(z)=\gamma_{\lambda}^{2}$, $K_{T}(z) / E_{T}(z)=\gamma_{T}^{2}$

$F(t)$ function describing the time dependence of the irradiance. (For a rotating body $F(t)$ is a square wave with period $P: F(t)=1$ when $0 \leq t \leq P / 2$, and $F(t)=0$ when $P / 2<t<P$.)

$G_{\lambda}\left(z, \Omega^{\prime}, \Omega\right), G_{v}\left(z, \Omega^{\prime}, \Omega\right), G_{T}\left(z, \Omega^{\prime}, \Omega\right)$ spectral visible, and thermal volume angular scattering coefficients equal to $\Sigma_{j} N_{j}(z) \sigma_{j} Q_{S \lambda j} p_{\lambda j}\left(\Omega^{\prime}, \Omega\right)$, $\sum_{j} N_{j}(z) \sigma_{j} Q_{S_{i j}} p_{v j}\left(\Omega^{\prime}, \Omega\right)$, and $\sum_{j} N_{j}(z) \sigma_{j} Q_{S T j} p_{T j}\left(\Omega^{\prime}, \Omega\right)$ respectively, for scattering radiation traveling in direction $\Omega^{\prime}$ into direction $\Omega$.

$g$ phase angle, equal to angle between the directions to the source and detector as seen from the medium. $g^{\prime}$ angle between the direction $\pi-\Omega^{\prime}$ from which the radiance comes and the direction $\Omega$ into which it is scattered.

$H(w, x) \quad(1+2 x) /(1+\sqrt{1-w} x)$.

$H_{1}(w)$ first moment of the $H$ functions.

$h_{0}$ Planck's constant.

$I_{\lambda}(z, \Omega, t), I_{v}(z, \Omega, t), I_{T}(z, \Omega, t)$ spectral, visible, and thermal radiances (diffuse radiant power per unit area per unit solid angle), respectively.

$I_{y 1}(z, t), I_{T 1}(z, t)$ hemispherically averaged visible and thermal radiances traveling into the upward hemisphere.

$I_{v 2}(z, t) I_{T 2}(z, t)$ hemispherically averaged visible and thermal radiances traveling into the downward hemisphere.

$i(t)$ angle between $\Omega_{0}$ and $z$.

$J_{\lambda}, J_{v}$ spectral and visible irradiance, respectively (collimated radiant power per unit area perpendicular to the direction of propagation) incident on the upper surface of the medium; for planetary applications, $J_{\lambda}$ has the spectrum of a blackbody with a temperature of $5770 \mathrm{~K}$, and $J_{v}=\mathscr{S} / D^{2}$, where $\mathscr{S}$ is the solar constant, $\mathscr{S}=1360 \mathrm{~W} \mathrm{~m}^{-2}$ and $D$ is the distance to the sun in astronomical units.

$j$ subscript denoting particle type.

$K_{\lambda}(z), K_{v}(z), K_{T}(z)$ spectral, visible, and thermal volume absorption coefficients, equal to $\sum_{j} N_{j}(z) \sigma_{j} Q_{A \lambda j}, \sum_{j} N_{j}(z) \sigma_{j} Q_{A v j}$, and $\sum_{j} N_{j}(z) \sigma_{j} Q_{A T_{j}}$ respectively.

$k$ solid state thermal conductivity.

$k_{0}$ Boltzmann's constant.

$L_{E v}, L_{E T}$ visible and thermal extinction lengths, equal to $1 / E_{\vartheta}$ and $1 / E_{T}$ respectively.

$N_{j}(z)$ number of particles per unit volume of type $j$.

$P$ period of rotation of the body.

$p_{\lambda j}\left(\Omega^{\prime}, \Omega\right), p_{v j}\left(\Omega^{\prime}, \Omega\right), p_{T j}\left(\Omega^{\prime}, \Omega\right)$ spectral, visible, and thermal angular phase functions of a particle of type $j$, respectively; if the particles are randomly oriented, the particle phase functions depend only on $g^{\prime}$.

$p_{\lambda}\left(z, \Omega^{\prime}, \Omega\right), p_{v}\left(z, \Omega^{\prime}, \Omega\right), p_{T}\left(z, \Omega^{\prime}, \Omega\right)$ spectral, visible, and thermal average volume particle scattering functions, equal to $G_{\lambda}\left(z, \Omega^{\prime}, \Omega\right) / S_{\lambda}(z)$, $G_{v}\left(z, \Omega^{\prime}, \Omega\right) / S_{z}(z)$, and $G_{T}\left(z, \Omega^{\prime}, \Omega\right) / S_{T}(z)$

$Q_{A \lambda j}(z), Q_{A v j}(z), Q_{A T j}(z)$ spectral, visible, and thermal volume absorption efficiencies of a particle of type $j$.

$Q_{E \lambda j}(z), Q_{E v j}(z), Q_{E T j}(z)$ spectral, visible, and thermal volume extinction efficiencies of a particle of type $j$.

$Q_{S \lambda j}(z), Q_{S v j}(z), Q_{S T j}(z)$ spectral, visible, and thermal volume scattering efficiencies of a particle of type $j$.

$S_{\lambda}(z), S_{v}(z), S_{T}(z)$ spectral, visible, and thermal volume scattering coefficients equal to $\Sigma_{j} N_{j}(z) \sigma_{j} Q_{S \lambda j}$, $\sum_{j} N_{j}(z) \sigma_{j} Q_{S \tau j}$, and $\sum_{j} N_{j}(z) \sigma_{j} Q_{S T j}$ respectively.

$T(z, t)$ absolute temperature.

$T_{s}(t)$ surface temperature, equal to $T(0, t)$

$t$ time.

$U_{\lambda}(T)$ Planck function. 
$w_{\lambda}, w_{y}, w_{T}$ spectral, visible, and thermal volume-average single scattering albedos, equal to $S_{\lambda}(z) / E_{\lambda}(z), S_{\nu}(z) / E_{\nu}(z)$, and $S_{T}(z) / E_{T}(z)$.

$z$ vertical position (positive upward).

$\beta_{\lambda}, \beta_{v}, \beta_{T}$ spectral, visible, and thermal hemispherical asymmetry scattering parameters, respectively.

$\gamma_{\lambda}, \gamma_{7}, \gamma_{T}$ spectral, visible, and thermal albedo factors equal to, $\left(1-w_{\lambda}\right)^{1 / 2},\left(1-w_{\nu}\right)^{1 / 2}$, and $\left(1-w_{T}\right)^{1 / 2}$ respectively.

$\varphi_{\lambda}(z, t), \varphi_{v}(z, t), \varphi_{T}(z, t)$ spectral, visible, and thermal average isotropic radiant fluxes, respectively.

$\varphi_{\lambda s}(t), \varphi_{z s}(t), \varphi_{T S}(t) \varphi_{\lambda}(0, t), \varphi_{\nu}(0, t)$, and $\varphi_{T}(0, t)$ values of the spectral, visible, and thermal fluxes equal to, respectively, at the surface.

$\Delta \varphi_{\lambda}(z, t), \Delta \varphi_{z}(z, t), \Delta \varphi_{r}(z, t)$ spectral, visible, and thermal average radiant up-down flux differences, respectively.

$\phi$ filling factors, equal to $\rho / \rho_{s}$.

$\vartheta$ angle between $\Omega$ and the normal to the surface.

$\lambda$ wavelength.

$\mu \cos \mathrm{e}$.

$\mu_{0}(t) \quad \cos i(t)$.

$\rho$ bulk density.

$\rho_{s} \quad$ particle solid density.

$\sigma_{j}(z)$ mean geometrical cross-sectional area of a particle of type $j$.

$\sigma_{0}$ Stefan-Boltzmann constant.

$\tau_{\lambda}(z)=\int_{z}^{\infty} E_{\lambda}\left(z^{\prime}\right) d z, \tau_{v}(z)=\int_{z}^{\infty} E_{v}\left(z^{\prime}\right) d z$, $\tau_{T}(z)=\int_{z}^{\infty} E_{T}\left(z^{\prime}\right) d z$ spectral, visible, and thermal optical depths, respectively.

$d \tau_{\lambda}-E_{\lambda}(z) d z, d \tau_{v}=-E_{y}(z) d z$, $d \tau_{T}=-E_{T}(z) d z$.

$\Omega$ direction into which the radiance is traveling through an element of volume within the medium.

$\Omega_{0} \quad$ direction toward the source from the medium (the incident irradiance travels into the direction $\pi-\Omega_{0}$ ).

$\zeta_{\lambda}=\left(1-\beta_{\lambda} w_{\lambda}\right)^{1 / 2}, \zeta_{v}=\left(1-\beta_{v} w_{v}\right)^{1 / 2}$, $\zeta_{T}=\left(1-\beta_{T} w_{T}\right)^{1 / 2}$, spectral, visible, and thermal asymmetry factors, respectively.

The following relations also hold:

$$
\begin{gathered}
\varepsilon_{\lambda j}=Q_{A \lambda j} \quad \varepsilon_{T j}=Q_{A T j} . \\
\mathscr{E}_{\lambda}(z)=K_{\lambda}(z) \quad \mathscr{E}_{T}(z)=K_{T}(z) . \\
Q_{S \lambda j}+Q_{A \lambda j}=Q_{E \lambda j}, Q_{S v j}+Q_{A v j}=Q_{E v j}, Q_{S T j}+Q_{A T j}=Q_{E T j} . \\
S_{\lambda}+K_{\lambda}=E_{\lambda}, \quad S_{v}+K_{v}=E_{v}, \quad S_{T}+K_{T}=E_{T} .
\end{gathered}
$$

$$
\gamma_{\lambda}^{2}=K_{\lambda} / E_{\lambda}, \quad \gamma_{v}^{2}=K_{v} / E_{v}, \quad \gamma_{T}^{2}=K_{T} / E_{T} .
$$

If the filling factor $\phi$ of the medium is not small, $N_{j}(z)$ must be multiplied by the factor $-\ln (1-\phi) / \phi$.

Acknowledgments. This work benefited from constructive suggestions by Paul Helfenstein and an anonymous reviewer. It is sponsored by a grant from the Planetary Geology and Geophysics Program, Solar System Exploration Division, National Aeronautics and Space Administration.

\section{References}

Brown, R. H., and D. Matson, Thermal effects of insolation propagation into the regoliths of airless bodies, Icarus, 72, 84-94, 1987.

Carrier, W., J. Mitchell, and A. Mahmood, The relative density of lunar soil, Proc. Lunar Sci. Conf., 4th, 2403-2411, 1973.

Conel, J., Infrared emissivities of silicates: Experimental results and a cloudy atmosphere model of spectral emission from condensed particulate medium, J. Geophys. Res., 74, 1614-1634, 1969.

Fountain, J., and E. West, Thermal conductivity of particulate basalt as a function of density in simulated lunar and Martian environments, J. Geophys. Res., 75, 4063-4070, 1970.

Goguen, J., A quantitative test of the applicability of independent scattering to high albedo planetary regoliths, Icarus, in press, 1996.

Hapke, B., Bidirectional reflectance spectroscopy, 1, Theory, J. Geophys. Res., 86, 3039-3054, 1981.

Hapke, B., Theory of Reflectance and Emittance Spectroscopy, Cambridge Univ. Press, New York, 1993a.

Hapke, B., Combined theory of reflectance and emittance spectroscopy, in Remote Geochemical Analysis, edited by C. Pieters and P. Englert, pp. 31-42, Cambridge Univ. Press, New York, $1993 \mathrm{~b}$.

Hapke, B., Applications of an energy transfer model to three problems in planetary regoliths: The solid-state greenhouse, thermal beaming and emittance spectra, J. Geophys. Res., this issue.

Henderson, B., and B. Jakosky, Near-surface thermal gradients and their effects on mid-infrared emission spectra of planetary surfaces, J. Geophys. Res., 99, 19,063-19,074, 1994.

Horai, K., and G. Simmons, Thermal property measurements on lunar material, in Thermal Characteristics of the Moon, edited by J. Lucas, pp. 243-268, MIT Press, Cambridge, Mass., 1972.

McKay, D., R. Fruland, and G. Heiken, Grain size and the evolution of lunar soils, Proc. Lunar Sci. Conf., 5th, 887-906, 1974.

Moersch, J., and P. Christensen, Thermal emission from particulate surfaces: A comparison of scattering models with measured spectra, J. Geophys. Res., 100, 7465-7477, 1995.

Salisbury, J., A. Wald, and D. D'Aria, Thermal-infrared remote sensing and Kirchhoff's law, 1, Laboratory measurement, J. Geophys. Res., 99, 11,897-11,911, 1994.

Spencer, J., A rough-surface thermosphysical model for airless planets, Icarus, 83, 27-38, 1990.

Wesselink, A., Heat conductivity and nature of the lunar surface material, Bull. Astron. Inst. Netherlands, 10, 351-363, 1948.

B. Hapke, 321 Old Engineering Hall, University of Pittsburgh, Pittsburgh, PA 15260. (e-mail: hapke@vms.cis.pitt.edu)

(Received May 9, 1995; revised March 18, 1996; accepted March 20, 1996.) 
